\title{
Closed Form Moment Formulae for the Lognormal SABR Model and Applications to Calibration Problems
}

\author{
Lorella Fatone $^{1}$, Francesca Mariani ${ }^{2}$, Maria Cristina Recchioni ${ }^{3}$, Francesco Zirilli ${ }^{4}$ \\ ${ }^{1}$ Dipartimento di Matematica e Informatica Università di Camerino via Madonna delle Carceri 9, Camerino, Italy \\ ${ }^{2}$ Dipartimento di Scienze Economiche Università degli Studi di Verona Vicolo Campo_ore 2, Verona, Italy \\ ${ }^{3}$ Dipartimento di Management Università Politecnica delle Marche Piazza Martelli 8, Ancona, Italy \\ ${ }^{4}$ Dipartimento di Matematica “G. Castelnuovo” Università di Roma “La Sapienza” Piazzale Aldo Moro 2, Roma, Italy \\ Email: lorella.fatone@unicam.it, francesca.mariani@univr.it,m.c.recchioni@univpm.it, zirilli@mat.uniroma1.it
}

Received August 11, 2013; revised September 17, 2013; accepted September 30, 2013

Copyright (c 2013 Lorella Fatone et al. This is an open access article distributed under the Creative Commons Attribution License, which permits unrestricted use, distribution, and reproduction in any medium, provided the original work is properly cited.

\begin{abstract}
We study two calibration problems for the lognormal SABR model using the moment method and some new formulae for the moments of the logarithm of the forward prices/rates variable. The lognormal SABR model is a special case of the SABR model [1]. The acronym "SABR" means "Stochastic- $\alpha \beta \rho$ " and comes from the original names of the model parameters (i.e., $\alpha, \beta, \rho$ ) [1]. The SABR model is a system of two stochastic differential equations widely used in mathematical finance whose independent variable is time and whose dependent variables are the forward prices/rates and the associated stochastic volatility. The lognormal SABR model corresponds to the choice $\beta=1$ and depends on three quantities: the parameters $\alpha, \rho$ and the initial stochastic volatility. In fact the initial stochastic volatility cannot be observed and can be regarded as a parameter. A calibration problem is an inverse problem that consists in determineing the values of these three parameters starting from a set of data. We consider two different sets of data, that is: i) the set of the forward prices/rates observed at a given time on multiple independent trajectories of the lognormal SABR model, ii) the set of the forward prices/rates observed on a discrete set of known time values along a single trajectory of the lognormal SABR model. The calibration problems corresponding to these two sets of data are formulated as constrained nonlinear least-squares problems and are solved numerically. The formulation of these nonlinear least-squares problems is based on some new formulae for the moments of the logarithm of the forward prices/rates. Note that in the financial markets the first set of data considered is hardly available while the second set of data is of common use and corresponds simply to the time series of the observed forward prices/rates. As a consequence the first calibration problem although realistic in several contexts of science and engineering is of limited interest in finance while the second calibration problem is of practical use in finance (and elsewhere). The formulation of these calibration problems and the methods used to solve them are tested on synthetic and on real data. The real data studied are the data belonging to a time series of exchange rates between currencies (euro/U.S. dollar exchange rates).
\end{abstract}

Keywords: SABR Model; Calibration Problems; FX Data

\section{Introduction}

We study two calibration problems for the lognormal SABR model using the moment method and some new formulae for the moments of the logarithm of the forward prices/rates variable. The lognormal SABR model is a special case of the "Stochastic- $\alpha \beta \rho$ " model which has become known under the acronym of SABR model [1]. The SABR model is widely used in the theory and practice of mathematical finance, for example, it is widely used to price interest rates derivatives and options on currencies exchange rates.
Let $t$ be a real variable that denotes time and $x_{t}, v_{t}$, $t>0$, be real stochastic processes that describe, respectively, the forward prices/rates and the associated stochastic volatility, as a function of time. The SABR model [1] assumes that the dynamics of the stochastic processes $x_{t}, v_{t}, t>0$, is defined by the following system of stochastic differential equations:

$$
\begin{aligned}
& \mathrm{d} x_{t}=\left|x_{t}\right|^{\beta} v_{t} \mathrm{~d} W_{t}, t>0, \\
& \mathrm{~d} v_{t}=\varepsilon v_{t} \mathrm{~d} Q_{t}, t>0,
\end{aligned}
$$

with the initial conditions: 


$$
\begin{aligned}
& x_{0}=\tilde{x}_{0}, \\
& v_{0}=\tilde{v}_{0},
\end{aligned}
$$

where $\beta \in[0,1]$ is the $\beta$-volatility and $\varepsilon>0$ is the volatility of volatility. Note that in the original paper [1] the volatility of volatility $\varepsilon$ was called $\alpha$. The stochastic processes $W_{t}, Q_{t}, t>0$, are standard Wiener processes such that $W_{0}=Q_{0}=0, \mathrm{~d} W_{t}, \mathrm{~d} Q_{t}$, $t>0$, are their stochastic differentials and we assume that:

$$
\left\langle\mathrm{d} W_{t} \mathrm{~d} Q_{t}\right\rangle=\rho \mathrm{d} t, t>0
$$

where $\langle\cdot\rangle$ denotes the expected value of $\cdot$ and $\rho \in(-1,1)$ is a constant known as correlation coefficient. The initial conditions $\tilde{x}_{0}, \tilde{v}_{0}$ are random variables that are assumed to be concentrated in a point with probability one. For simplicity, we identify these random variables with the points where they are concentrated. We assume $\tilde{v}_{0}>0$ (with probability one) so that Equation (2) implies that $v_{t}>0$ (with probability one) for $t>0$. Note that the initial stochastic volatility $\tilde{v}_{0}$ and the stochastic volatility $v_{t}, t>0$, cannot be observed in the financial markets. That is, $\tilde{v}_{0}$ must be regarded as a parameter of the model together with $\beta$, $\varepsilon$ and $\rho$.

The value of the parameter $\beta \in[0,1]$ determines the forward prices/rates process, that is, it determines Equation (1). The most common choices of $\beta$ are: $\beta=0, \beta=1 / 2$ and $\beta=1$.

Setting $\beta=0$ in (1) the forward prices/rates process reduces to:

$$
\mathrm{d} x_{t}=v_{t} \mathrm{~d} W_{t}, t>0 .
$$

The corresponding model (6), (2), (3), (4) is known as the normal SABR model. This model has a forward prices/rates process whose increments are stochastic normally distributed, that is, the increments are normally distributed with mean zero and a stochastic standard deviation lognormally distributed. This permits to the forward prices/rates $x_{t}, t>0$, to become negative. Usually this is not a desirable property. In fact, in financial applications most of the times prices/rates are supposed to be positive. However, in some anomalous circumstances negative quantities such as negative interest rates can be considered.

The choice $\beta=1 / 2$ in (1) gives the following forward prices/rates process:

$$
\mathrm{d} x_{t}=\sqrt{\left|x_{t}\right|} v_{t} \mathrm{~d} W_{t}, t>0 .
$$

The model (7), (2), (3), (4) can be seen as a stochastic volatility version of the CIR model with no drift. The CIR model is a short term interest rate model introduced by Cox, Ingersoll and Ross (CIR) in [2]. In the CIR model the volatility $v_{t}, t>0$, is a constant, that is, $v_{t}=\tilde{v}_{0}, t>0$. Note that the model (7), (2), (3), (4) reduces to the CIR model (with no drift) when $\varepsilon=0$. When $\varepsilon>0$ the volatility is governed by (2). In the SABR model (7), (2) when the initial conditions (3), (4) are positive (with probability one) negative forward prices/rates can be avoided.

Finally, the choice $\beta=1$ in (1) produces:

$$
\mathrm{d} x_{t}=\left|x_{t}\right| v_{t} \mathrm{~d} W_{t}, t>0
$$

The model (8), (2), (3), (4) is known as lognormal SABR model. It is a stochastic volatility version of the Black model. The Black model is a special case of the Black-Scholes model [3] obtained when the drift parameter of the Black-Scholes model is equal to zero. In the Black model the underlying asset price is modeled as a geometric Brownian motion. Unlike in the Black model, where the volatility is a constant, in the lognormal SABR model the volatility is a stochastic process itself (see (2)). Note that model (8), (2), (3), (4) reduces to the Black model when $\varepsilon=0$. In the lognormal SABR model the positivity (with probability one) of the forward prices/ rates $x_{t}$ is guaranteed for $t>0$ when the initial conditions (3), (4) are positive (with probability one). In particular when the initial conditions (3), (4) are positive (with probability one) the absolute value in (8) can be removed.

The choice made in this paper of studying the lognormal SABR model is motivated by the fact that the lognormal model is the most used SABR model in the practice of the financial markets. Moreover, after the normal SABR model (that has been studied in [4]) the lognormal SABR model is mathematically the simplest model in the class of the SABR models (1)-(4).

Note that in the SABR model the forward prices/rates random variable is represented as a compound random variable and that the SABR model can be seen as a stochastic state space model [5]. Compound random variables and state space models are widely used in science and engineering. This means that the methods and the results presented here to study the lognormal SABR model can be extended outside mathematical finance to a wide class of problems.

In this paper we concentrate on the study of the lognormal SABR model (8), (2), (3), (4), i.e., in (1) we choose $\beta=1$, and we study the calibration problem for this model. That is, we study the problem of determining the unknown parameters $\varepsilon, \rho, \tilde{v}_{0}$ of the lognormal SABR model starting from the knowledge of a set of data. The sets of data considered are: i) the set of the forward prices/rates observed at a given time on multiple independent trajectories of the lognormal SABR model, ii) the set of the forward prices/rates observed on a discrete set of known time values along a single trajectory of the 
lognormal SABR model. The formulation of the calibration problems corresponding to these two sets of data is based on some new closed form formulae for the moments of the logarithm of the forward prices/rates variable. Using these formulae the calibration problems considered are formulated as constrained nonlinear leastsquares problems. The moments formulae are deduced extending to the lognormal SABR model a method introduced in [4] in the study of the normal SABR model.

Note that the data set used in the first calibration problem, that is, a data sample made of observations at a given time on multiple trajectories, is hardly available in the financial markets. In fact, in the financial markets usually it is not possible to repeat the "experiment" as done routinely in contexts where observations are made in experiments carried out in a laboratory. This implies that the first calibration problem although realistic in several fields of science and engineering has limited applications in finance. Instead, the second calibration problem is of practical use in finance since single trajectory data samples are easily available in the financial markets and can be identified with time series of observed forward prices/rates.

An alternative approach to study the calibration problem for the lognormal SABR model corresponding to the single trajectory data sample consists in extending the method proposed in $[6,7]$ to study a similar calibration problem for the Heston model and for some of its variations. This method is based on the idea of maximizing a likelihood function. However, the use of closed form moment formulae (see Formulae (65)-(68)) rather than the use of a likelihood function involving the transition probability density function of the differential model and the solution of a kind of Kushner equation (see [6,7]) gives to the method based on the moment formulae presented here a substantial computational advantage in comparison to the method suggested in [6], [7]. A similar statement holds when the method presented here is compared to methods where averages of quantities implicitly defined by the differential model (such as the moments) are computed using statistical simulation.

The numerical solution of the nonlinear least-squares problems that translate the calibration problems considered can greatly benefit from the availability of a good initial guess to initialize the optimization algorithm. In Section 3 we discuss briefly how to exploit the first moment formula obtained in Section 2 to build the initial guesses needed.

Note that, extending the results presented in [4], it is possible to define ad hoc statistical tests that can be used to associate a statistical significance level to the parameter values obtained as solution of the calibration problems. We do not consider statistical tests and statistical significance levels in this paper.
The remainder of the paper is organized as follows. In Section 2, new formulae for some of the moments of the logarithm of the forward prices/rates variable of the lognormal SABR model are derived. In Section 3, the calibration problems for the lognormal SABR model corresponding to the two data sets discussed previously are formulated as constrained nonlinear least-squares problems. Finally, in Section 4 we solve numerically the calibration problems presented in Section 3 and we discuss the results obtained in numerical experiments on synthetic and on real data. The real data studied are time series of euro/U.S. dollar exchange rates.

\section{Formulae for the Moments of the Lognormal SABR Model}

Let us deduce closed form formulae for the moments of the logarithm of forward prices/rates of the lognormal SABR model. Let us consider the model given by:

$$
\begin{gathered}
\mathrm{d} x_{t}=x_{t} v_{t} \mathrm{~d} W_{t}, t>0, \\
\mathrm{~d} v_{t}=\varepsilon v_{t} \mathrm{~d} Q_{t}, t>0,
\end{gathered}
$$

with positive initial conditions (3), (4) and the assumption (5). That is, we assume $\tilde{x}_{0}>0$ (with probability one) and $\tilde{v}_{0}>0$ (with probability one), so that Equations (9), (10) imply $x_{t}>0$ (with probability one), and $v_{t}>0$ (with probability one) for $t>0$.

Let $\xi_{t}=\ln \left(x_{t}\right), t>0$, be the logarithm of the forward prices/rates. Using the variables $\xi_{t}, v_{t}, t>0$, the stochastic differential Equations (9), (10) and the initial conditions (3), (4) are rewritten as follows:

$$
\begin{gathered}
\mathrm{d} \xi_{t}=-\frac{1}{2} v_{t}^{2} \mathrm{~d} t+v_{t} \mathrm{~d} W_{t}, t>0, \\
\mathrm{~d} v_{t}=\varepsilon v_{t} \mathrm{~d} Q_{t}, t>0, \\
\xi_{0}=\tilde{\xi}_{0}=\ln \left(\tilde{x}_{0}\right), \\
v_{0}=\tilde{v}_{0} .
\end{gathered}
$$

Starting from the expression obtained in [8] for the transition probability density function of the stochastic processes $\xi_{t}, v_{t}, t>0$, we deduce explicit formulae (eventually involving integrals) for the moments with respect to zero of $\xi_{t}, v_{t}, t>0$, and of $x_{t}, v_{t}, t>0$. In particular, we derive closed form formulae (that do not involve integrals) for the first five moments with respect to zero of $\xi_{t}, t>0$.

In [8], using the backward Kolmogorov Equation associated to (11), (12), the following formula for the transition probability density function $p_{L}$ of the stochastic processes $\xi_{t}, v_{t}, t>0$, implicitly defined by (11)-(14) has been obtained: 


$$
\begin{aligned}
& p_{L}\left(\xi, v, t, \xi^{\prime}, v^{\prime}, t^{\prime}\right) \\
& =\frac{1}{2 \pi} \int_{-\infty}^{+\infty} d k \mathrm{e}^{-\imath k\left(\xi^{\prime}-\xi\right)} g_{L}\left(t-t^{\prime}, k, v, v^{\prime}, \varepsilon, \rho\right), \\
& \quad(\xi, v),\left(\xi^{\prime}, v^{\prime}\right) \in \mathbb{R} \times \mathbb{R}^{+}, t, t^{\prime} \geq 0, t-t^{\prime}>0,
\end{aligned}
$$

where $\imath$ is the imaginary unit and we have $\xi_{t}=\xi$, $v_{t}=v, \xi_{t^{\prime}}=\xi^{\prime}, v_{t^{\prime}}=v^{\prime}, t, t^{\prime} \geq 0, t-t^{\prime}>0$. In (15), when $t^{\prime}=0$ we must choose $\xi^{\prime}=\tilde{\xi}_{0}, \quad v^{\prime}=\tilde{v}_{0}$. The function $g_{L}$ is given by:

$$
\begin{gathered}
g_{L}\left(s, k, v, v^{\prime}, \varepsilon, \rho\right)=\frac{2}{\pi^{2}} \mathrm{e}^{-\frac{s}{8} \varepsilon^{2}}\left(\frac{\sqrt{v^{\prime}}}{v \sqrt{v}}\right) \mathrm{e}^{\imath k \frac{\rho\left(v^{\prime}-v\right)}{\varepsilon}} \\
\times \int_{0}^{+\infty} \mathrm{d} \omega \mathrm{e}^{-\tau \omega^{2}} \omega \sinh (\pi \omega) K_{\imath \omega}(\varsigma(k) v) K_{\iota \omega}\left(\varsigma(k) v^{\prime}\right), \\
s \in \mathbb{R}^{+}, k \in \mathbb{R}, v, v^{\prime} \in \mathbb{R}^{+}, \varepsilon>0, \rho \in(-1,1),
\end{gathered}
$$

where the function $K_{\eta}$ denotes the second type modified Bessel function of order $\eta$ (see [9] p. 5). Finally, $\varsigma^{2}(k), k \in \mathbb{R}$, is defined as

$$
\varsigma^{2}(k)=\frac{k^{2}}{\varepsilon^{2}}\left(1-\rho^{2}\right)-\mathrm{i} \frac{k}{\varepsilon^{2}}, k \in \mathbb{R} .
$$

The function $g_{L}$ can be rewritten as follows [8]:

$$
\begin{aligned}
& g_{L}\left(s, k, v, v^{\prime}, \varepsilon, \rho\right)=\frac{\sqrt{v^{\prime}}}{\pi \sqrt{\pi}} \mathrm{e}^{-\frac{s}{8} \varepsilon^{2}} \frac{1}{v \sqrt{v}} \mathrm{e}^{\imath \frac{\rho\left(v^{\prime}-v\right)}{\varepsilon}} \\
& \times \frac{\mathrm{e}^{\frac{\pi^{2}}{2 s \varepsilon^{2}}}}{\sqrt{2 s \varepsilon^{2}}} \int_{0}^{+\infty} \mathrm{d} u \sinh (u) \sin \left(\frac{\pi u}{s \varepsilon^{2}}\right) \mathrm{e}^{-u^{2} /\left(2 s \varepsilon^{2}\right)} \\
& \times \int_{0}^{+\infty} \mathrm{d} y \mathrm{e}^{2{ }^{2} \frac{v v^{\prime} k}{2 y \varepsilon^{2}}} \mathrm{e}^{-y\left(v^{\prime 2}+v^{2}+2 v v^{\prime} \cosh (u)\right) /\left(2 v v^{\prime}\right)} \mathrm{e}^{-\frac{v^{\prime}\left(1-\rho^{2}\right) k^{2}}{2 \varepsilon^{2} y}}, \\
& s \in \mathbb{R}^{+}, k \in \mathbb{R}, v, v^{\prime} \in \mathbb{R}^{+}, \varepsilon>0, \rho \in(-1,1) .
\end{aligned}
$$

Formulae (15), (16) or (15), (18) give $p_{L}$ as a two dimensional integral of an explicitly known integrand. Note that in (15) the transition probability density function $p_{L}$ is written using the variables $\xi_{t}=\ln \left(x_{t}\right)$, $v_{t}, t>0$. It is easy to obtain a formula analogous to formula (15) for the transition probability density function written in the original variables $x_{t}, v_{t}, t>0$.

Formulae (15), (16) and (15), (18) are representation formulae for $p_{L}$ that hold when $\rho \in(-1,1)$. These formulae have been obtained in [8]. Previously when $\rho \neq 0$ for $p_{L}$ only series expansions in powers of $\rho$ with base point $\rho=0$ were known (see, for example, $[10,11]$ and the references therein).

Let us begin deriving some formulae for the moments $\mathcal{M}_{n, m}, n, m=0,1, \cdots$, with respect to zero of the variables $x_{t}, v_{t}, t>0$, of the lognormal SABR model (9), (10), (3), (4), namely,

$$
\begin{gathered}
\mathcal{M}_{n, m}\left(t, \xi^{\prime}, v^{\prime}, t^{\prime}\right)=\int_{-\infty}^{+\infty} \mathrm{d} \xi \mathrm{e}^{n \xi} \int_{0}^{+\infty} \mathrm{d} v v^{m} p_{L}\left(\xi, v, t, \xi^{\prime}, v^{\prime}, t^{\prime}\right), \\
\xi^{\prime} \in \mathbb{R}, v^{\prime} \in \mathbb{R}^{+}, t, t^{\prime} \geq 0, t-t^{\prime}>0, m, n=0,1, \cdots .
\end{gathered}
$$

We distinguish two cases, that is: the case $n=0$ and the case $n>0$.

When $n=0$ we have:

$$
\begin{aligned}
& \mathcal{M}_{0, m}\left(t, \xi^{\prime}, v^{\prime}, t^{\prime}\right)=\int_{-\infty}^{+\infty} \mathrm{d} \xi \int_{0}^{+\infty} \mathrm{d} v v^{m} p_{L}\left(\xi, v, t, \xi^{\prime}, v^{\prime}, t^{\prime}\right) \\
& =\int_{-\infty}^{+\infty} \mathrm{d} k \delta(k) \mathrm{e}^{-\imath k \xi^{\prime}}\left\{\int_{0}^{+\infty} \mathrm{d} v v^{m} g_{L}\left(t-t^{\prime}, k, v, v^{\prime}, \varepsilon, \rho\right)\right\} \\
& =\int_{0}^{+\infty} \mathrm{d} v v^{m} g_{L}\left(t-t^{\prime}, 0, v, v^{\prime}, \varepsilon, \rho\right), \\
& \quad \xi^{\prime} \in \mathbb{R}, v^{\prime} \in \mathbb{R}^{+}, t, t^{\prime} \geq 0, t-t^{\prime}>0, m=0,1, \cdots,
\end{aligned}
$$

where $\delta$ is the Dirac's delta. From (18) we have:

$$
\begin{aligned}
& \int_{0}^{+\infty} \mathrm{d} v v^{m} g_{L}\left(s, 0, v, v^{\prime}, \varepsilon, \rho\right) \\
& =\frac{\sqrt{v^{\prime}}}{\pi \sqrt{\pi}} \mathrm{e}^{-\frac{s}{8} \varepsilon^{2}} \frac{\mathrm{e}^{\frac{\pi^{2}}{2 s \varepsilon^{2}}}}{\sqrt{2 s \varepsilon^{2}}} \int_{0}^{+\infty} \mathrm{d} u \sinh (u) \sin \left(\frac{\pi u}{s \varepsilon^{2}}\right) \mathrm{e}^{-u^{2} /\left(2 s \varepsilon^{2}\right)} \\
& \quad \times \int_{0}^{+\infty} \mathrm{d} v v^{m-3 / 2} \int_{0}^{+\infty} \mathrm{d} y \mathrm{e}^{-y \frac{v}{2 v^{\prime}}} \mathrm{e}^{-y \frac{v^{\prime}}{2 v}} \mathrm{e}^{-y \cosh u}, \\
& \quad s, v^{\prime} \in \mathbb{R}^{+}, \varepsilon>0, \rho \in(-1,1), m=0,1, \cdots .
\end{aligned}
$$

Using formula (29) on page 146 of [12] we have:

$$
\begin{aligned}
& \int_{0}^{+\infty} \mathrm{d} v v^{\mu-3 / 2} \mathrm{e}^{-y \frac{v}{2 v^{\prime}}} \mathrm{e}^{-y \frac{v^{\prime}}{2 v}}=2\left(v^{\prime}\right)^{\mu-1 / 2} K_{\mu-1 / 2}(y), \\
& v^{\prime} \in \mathbb{R}^{+}, y \in \mathbb{R}^{+}, \mu \in \mathbb{R},
\end{aligned}
$$

and this implies that:

$$
\begin{aligned}
\mathcal{M}_{0, m}\left(t, \xi^{\prime}, v^{\prime}, t^{\prime}\right) & =\frac{2 v^{\prime m}}{\pi \sqrt{\pi}} \mathrm{e}^{-\frac{s}{8} \varepsilon^{2}} \frac{\mathrm{e}^{\frac{\pi^{2}}{2 s \varepsilon^{2}}}}{\sqrt{2 s \varepsilon^{2}}} \\
& \times \int_{0}^{+\infty} \mathrm{d} u \sinh (u) \sin \left(\frac{\pi u}{s \varepsilon^{2}}\right) \mathrm{e}^{-u^{2} /\left(2 s \varepsilon^{2}\right)} \\
& \times \int_{0}^{+\infty} \mathrm{d} y K_{m-1 / 2}(y) \mathrm{e}^{-y \cosh u}, \\
& \xi^{\prime} \in \mathbb{R}, v^{\prime} \in \mathbb{R}^{+}, t, t^{\prime} \geq 0, \\
& t-t^{\prime}>0, m=0,1, \cdots
\end{aligned}
$$

From Formula (24) on page 197 of [12] it follows that:

$$
\int_{0}^{+\infty} \mathrm{d} y K_{ \pm 1 / 2}(y) \mathrm{e}^{-y \cosh u}=\pi \frac{\sinh (u / 2)}{\sinh (u)}, u \in \mathbb{R} .
$$

From Formulaes (23) and (24) and using formula (37) on page 92 of [12] we can conclude that $\mathcal{M}_{0,0}\left(t, \xi^{\prime}, v^{\prime}, t^{\prime}\right)=1, \quad \xi^{\prime} \in \mathbb{R}, \quad v^{\prime} \in \mathbb{R}^{+}, t, t^{\prime} \geq 0, t-t^{\prime}>0$ and that $\mathcal{M}_{0,1}\left(t, \xi^{\prime}, v^{\prime}, t^{\prime}\right)=v^{\prime}, \quad \xi^{\prime} \in \mathbb{R}$, $v^{\prime} \in \mathbb{R}^{+}, t, t^{\prime} \geq 0, t-t^{\prime}>0$. Note that the moments $\mathcal{M}_{0, m}, m=2,3, \cdots$, diverge. In fact, when $n=0$ integrating (20) first with respect to $k$ when $k \in \mathbb{R}$, and then with respect to $\xi$ when $\xi \in \mathbb{R}$, we have: 


$$
\begin{aligned}
\mathcal{M}_{0, m}\left(t, \xi^{\prime}, v^{\prime}, t^{\prime}\right)= & \frac{1}{2 \pi} \int_{-\infty}^{+\infty} \mathrm{d} \xi \int_{0}^{+\infty} \mathrm{d} v v^{m} \int_{-\infty}^{+\infty} \mathrm{d} k \mathrm{e}^{\imath k\left(\xi-\xi^{\prime}\right)} g_{L}\left(t-t^{\prime}, k, v, v^{\prime}, \varepsilon, \rho\right) \\
= & 2 \frac{v^{\prime} \sqrt{v^{\prime}}}{\pi \sqrt{\pi}} \times \mathrm{e}^{-\frac{s}{8} \varepsilon^{2}} \frac{\mathrm{e}^{\frac{\pi^{2}}{2 s \varepsilon^{2}}}}{\sqrt{2 s \varepsilon^{2}}} \int_{0}^{+\infty} \mathrm{d} u \sinh (u) \sin \left(\frac{\pi u}{s \varepsilon^{2}}\right) \mathrm{e}^{\frac{-u^{2}}{2 s \varepsilon^{2}}} \\
& \times \int_{0}^{+\infty} \mathrm{d} v v^{m-1 / 2} \frac{1}{v^{2}+v^{\prime 2}+2 v v^{\prime} \cosh (u)}, \xi^{\prime} \in \mathbb{R}, v^{\prime} \in \mathbb{R}^{+}, t, t^{\prime} \geq 0, t-t^{\prime}>0, m=0,1, \cdots
\end{aligned}
$$

Formula (25) is equivalent to Formula (23) and shows that in order to have a convergent integral we must choose $-1 / 2<m<3 / 2$.
When $n>0$ using Formulaes (15) and (18) in the definition (19) we have:

$$
\begin{aligned}
\mathcal{M}_{n, m}\left(t, \xi^{\prime}, v^{\prime}, t^{\prime}\right)= & \frac{1}{2 \pi} \int_{-\infty}^{+\infty} \mathrm{d} \xi \mathrm{e}^{n \xi} \int_{0}^{+\infty} \mathrm{d} v v^{m} \times \int_{-\infty}^{+\infty} \mathrm{d} k \mathrm{e}^{\imath k\left(\xi-\xi^{\prime}\right)} g_{L}\left(t-t^{\prime}, k, v, v^{\prime}, \varepsilon, \rho\right) \\
= & -2 \frac{\mathrm{e}^{n \xi^{\prime}} v^{\prime} \sqrt{v^{\prime}}}{\pi \sqrt{\pi}} \sqrt{\frac{n-n^{2}\left(1-\rho^{2}\right)}{\varepsilon^{2}}} \mathrm{e}^{-\frac{s}{8} \varepsilon^{2}} \frac{\mathrm{e}^{\frac{\pi^{2}}{2 s \varepsilon^{2}}}}{\sqrt{2 s \varepsilon^{2}}} \times \int_{0}^{+\infty} \mathrm{d} u \sinh (u) \sin \left(\frac{\pi u}{s \varepsilon^{2}}\right) \mathrm{e}^{-u^{2} /\left(2 s \varepsilon^{2}\right)} \\
& \times \int_{0}^{+\infty} \mathrm{d} v v^{m-1 / 2} \mathrm{e}^{-n \rho\left(v^{\prime}-v\right) / \varepsilon} \times \frac{\left(\frac{\sqrt{n-n^{2}\left(1-\rho^{2}\right)}}{\varepsilon} \sqrt{v^{2}+v^{\prime 2}+2 v v^{\prime} \cosh (u)}\right)}{\sqrt{v^{2}+v^{\prime 2}+2 v v^{\prime} \cosh (u)}} \\
& \xi^{\prime} \in \mathbb{R}, v^{\prime} \in \mathbb{R}^{+}, t, t^{\prime} \geq 0, t-t^{\prime}>0, n=1,2, \cdots, m=0,1, \cdots
\end{aligned}
$$

Note that for $n_{1}=1,2, \cdots$, when

$-1<\rho \leq-\sqrt{\left(n_{1}-1\right) / n_{1}} \quad$ or $\sqrt{\left(n_{1}-1\right) / n_{1}} \leq \rho<1 \quad$ the integrals appearing in (26) with $n=1,2, \cdots, n_{1}$, $m=0,1, \cdots$, and the corresponding moments are convergent. A similar existence condition for the moments of the lognormal SABR model has already been derived in [13], however in [13] no explicit integral representation formula for the moments like Formula (26) is given.

Note that when $n=1$ and $m=0$ formula (??) gives $\mathcal{M}_{1,0}\left(t, \xi^{\prime}, v^{\prime}, t^{\prime}\right)=\mathrm{e}^{\xi^{\prime}}, \quad \xi^{\prime} \in \mathbb{R}, v^{\prime} \in \mathbb{R}^{+}, t, t^{\prime} \geq 0, t-t^{\prime}>0$, when $\rho \in(-1,1)$. Recall that $x^{\prime}=\mathrm{e}^{\xi^{\prime}}, \xi^{\prime} \in \mathbb{R}$.

Let us consider now the moments $\mathcal{L}_{n, m}, n, m=0,1, \cdots$, with respect to zero of the variables $\xi_{t}, v_{t}, t>0$, defined by (11)-(14), i.e.

$$
\begin{gathered}
\mathcal{L}_{n, m}\left(t, \xi^{\prime}, v^{\prime}, t^{\prime}\right)=\int_{-\infty}^{+\infty} \mathrm{d} \xi \xi^{n} \int_{0}^{+\infty} \mathrm{d} v v^{m} p_{L}\left(\xi, v, t, \xi^{\prime}, v^{\prime}, t^{\prime}\right), \\
\xi^{\prime} \in \mathbb{R}, v^{\prime} \in \mathbb{R}^{+}, t, t^{\prime} \geq 0, t-t^{\prime}>0, m, n=0,1, \cdots .
\end{gathered}
$$

The procedure used here to calculate the moments (27) generalizes the procedure used in [4] to calculate the moments with respect to zero of the variables $x_{t}, v_{t}$, $t>0$, of the normal SABR model.

Substituting (15) into (27) and using the properties of the Fourier transform we have:

$$
\begin{aligned}
\mathcal{L}_{n, m}\left(t, \xi^{\prime}, v^{\prime}, t^{\prime}\right)= & \sum_{j=0}^{n}\left(\begin{array}{c}
n \\
j
\end{array}\right)\left(\xi^{\prime}\right)^{n-j} \frac{(-1)^{j}}{\imath^{j}} \times \int_{0}^{+\infty} \mathrm{d} v v^{m} \int_{-\infty}^{+\infty} \mathrm{d} k \delta(k) \frac{\mathrm{d}^{j}}{\mathrm{~d} k^{j}} g_{L}\left(t-t^{\prime}, k, v, v^{\prime} \varepsilon, \rho\right) \\
= & \sum_{j=0}^{n}\left(\begin{array}{c}
n \\
j
\end{array}\right)\left(\xi^{\prime}\right)^{n-j} \imath^{j} \times\left.\int_{0}^{+\infty} \mathrm{d} v v^{m} \frac{\mathrm{d}^{j}}{\mathrm{~d} k^{j}} g_{L}\left(t-t^{\prime}, k, v, v^{\prime} \varepsilon, \rho\right)\right|_{k=0}, \\
& \xi^{\prime} \in \mathbb{R}, v^{\prime} \in \mathbb{R}^{+}, t, t^{\prime} \geq 0, t-t^{\prime}>0, n, m=0,1, \cdots .
\end{aligned}
$$

Let us calculate the integrals contained in (28). For $j=0,1, \cdots$ let $G_{j}$ be the $j$-th order derivative with respect to $k$ of the function $g_{L}$ evaluated at $k=0$, that is, let $G_{j}\left(s, v, v^{\prime}\right)=\left(\mathrm{d}^{j} g_{L} / \mathrm{d} k^{j}\right)\left(s, 0, v, v^{\prime}\right)$, $s, v, v^{\prime} \in \mathbb{R}^{+}$. To simplify the notation we have omitted in the previous formula, and we will omit from now on, the dependence of $g_{L}$ and of $G_{j}, j=0,1, \cdots$, from $\varepsilon$ and $\rho$. Using the functions $G_{j}, j=0,1, \cdots$, Formula 
(28) can be rewritten as follows:

$$
\begin{aligned}
& \mathcal{L}_{n, m}\left(t, \xi^{\prime}, v^{\prime}, t^{\prime}\right) \\
& =\sum_{j=0}^{n}\left(\begin{array}{l}
n \\
j
\end{array}\right)\left(\xi^{\prime}\right)^{n-j} \imath^{j} \times \int_{0}^{+\infty} \mathrm{d} v v^{m} G_{j}\left(t-t^{\prime}, v, v^{\prime}\right), \\
& \xi^{\prime} \in \mathbb{R}, v^{\prime} \in \mathbb{R}^{+}, t, t^{\prime} \geq 0, t-t^{\prime}>0, m, n=0,1, \cdots .
\end{aligned}
$$

We restrict our attention to $\mathcal{L}_{n, 0}\left(t, \xi^{\prime}, v^{\prime}, t^{\prime}\right), \quad \xi^{\prime} \in \mathbb{R}$, $v^{\prime} \in \mathbb{R}^{+}, \quad t, t^{\prime} \geq 0, t-t^{\prime}>0, n=0,1, \cdots$. In fact, in Section 3 in the solution of the calibration problems we will use $\mathcal{L}_{n, 0}, \quad n=1,2,3,4$. The choice of considering $m=0$ in the moments used to solve the calibration problems is due to the fact that in the financial markets the variable $x_{t}, t>0$, and, as a consequence, the variable $\xi_{t}$, $t>0$, can be observed, while the variable $v_{t}, t>0$, cannot be observed. That is, the moments $\mathcal{L}_{n, m}$, $n=0,1, \cdots, m>0$, cannot be easily estimated from observed data, while the moments $\mathcal{L}_{n, 0}, n=0,1, \cdots$, can be estimated immediately from observed data.

Let us define

$$
\begin{aligned}
& \begin{aligned}
\mathcal{L}_{n}^{*}\left(s, \xi^{\prime}, v^{\prime}\right) & =\mathcal{L}_{n, 0}\left(t, \xi^{\prime}, v^{\prime}, t^{\prime}\right) \\
& =\sum_{j=0}^{n}\left(\begin{array}{c}
n \\
j
\end{array}\right)\left(\xi^{\prime}\right)^{n-j} \imath^{j} D_{j}\left(s, v^{\prime}\right),
\end{aligned} \\
& s=t-t^{\prime} \in \mathbb{R}^{+}, \xi^{\prime} \in \mathbb{R}, v^{\prime} \in \mathbb{R}^{+}, n=0,1, \cdots .
\end{aligned}
$$

where

$$
\begin{aligned}
& D_{j}\left(s, v^{\prime}\right)=\int_{0}^{+\infty} \mathrm{d} v G_{j}\left(s, v, v^{\prime}\right) \\
& =\left.\int_{0}^{+\infty} \mathrm{d} v \frac{\mathrm{d}^{j}}{\mathrm{~d} k^{j}} g_{L}\left(s, k, v, v^{\prime}\right)\right|_{k=0}, s, v^{\prime} \in \mathbb{R}^{+}, j=0,1, \cdots,
\end{aligned}
$$

and

$$
\begin{gathered}
\tilde{D}_{j}\left(s, k, v^{\prime}\right)=\frac{\mathrm{d}^{j}}{\mathrm{~d} k^{j}} \int_{0}^{+\infty} \mathrm{d} v g_{L}\left(s, k, v, v^{\prime}\right), \\
s \in \mathbb{R}^{+}, k \in \mathbb{R}, v^{\prime} \in \mathbb{R}^{+}, j=0,1, \cdots .
\end{gathered}
$$

We have

$$
\begin{aligned}
& D_{j}\left(s, v^{\prime}\right)=\left.\tilde{D}_{j}\left(s, k, v^{\prime}\right)\right|_{k=0} \\
& =\left.\left(\frac{\mathrm{d}^{j}}{\mathrm{~d} k^{j}} \int_{0}^{+\infty} \mathrm{d} v g_{L}\left(s, k, v, v^{\prime}\right)\right)\right|_{k=0}, \\
& s, v^{\prime} \in \mathbb{R}^{+}, j=0,1, \cdots .
\end{aligned}
$$

The functions $D_{j}, j=0,1, \cdots$, can be determined by solving the initial value problems deduced below. The function $g_{L}$ is the solution of the following initial value problem (see [8]):

$$
\begin{aligned}
\frac{\partial g_{L}}{\partial s}= & \frac{\varepsilon^{2}}{2} v^{\prime 2} \frac{\partial^{2} g_{L}}{\partial v^{\prime 2}}-\frac{k^{2}}{2} v^{\prime 2} g_{L}-\imath k \rho \varepsilon v^{\prime 2} \frac{\partial g_{L}}{\partial v^{\prime}} \\
& +\frac{1}{2} \imath k v^{\prime 2} g_{L}, \quad s \in \mathbb{R}^{+}, k \in \mathbb{R}, v^{\prime} \in \mathbb{R}^{+},
\end{aligned}
$$

$$
g_{L}\left(0, k, v, v^{\prime}\right)=\delta\left(v^{\prime}-v\right), k \in \mathbb{R}, v, v^{\prime} \in \mathbb{R}^{+} .
$$

Equation (34) is the Fourier transform of the backward Kolmogorov equation of the lognormal SABR model and the parameter $k \in \mathbb{R}$ that appears in (34) is the conjugate variable in the Fourier transform of the variable $\xi^{\prime}-\xi \in \mathbb{R}$.

Integrating both sides of (34), (35) with respect to $v$ when $v \in \mathbb{R}^{+}$we have:

$$
\begin{gathered}
\frac{\partial \tilde{D}_{0}}{\partial s}=\frac{\varepsilon^{2}}{2} v^{\prime 2} \frac{\partial^{2} \tilde{D}_{0}}{\partial v^{\prime 2}}-\frac{k^{2}}{2} v^{\prime 2} \tilde{D}_{0}-\imath k \rho \varepsilon v^{\prime 2} \frac{\partial D_{0}}{\partial v^{\prime}} \\
+\frac{1}{2} \imath k v^{\prime 2} \tilde{D}_{0}, \quad s \in \mathbb{R}^{+}, k \in \mathbb{R}, v^{\prime} \in \mathbb{R}^{+}, \\
\tilde{D}_{0}\left(0, k, v^{\prime}\right)=1, k \in \mathbb{R}, v^{\prime} \in \mathbb{R}^{+} .
\end{gathered}
$$

When $k=0$, Equations (36), (37) reduce to:

$$
\begin{gathered}
\frac{\partial D_{0}}{\partial s}=\frac{\varepsilon^{2}}{2} v^{\prime 2} \frac{\partial^{2} D_{0}}{\partial v^{\prime 2}}, s \in \mathbb{R}^{+}, k \in \mathbb{R}, v^{\prime} \in \mathbb{R}^{+}, \\
D_{0}\left(0, v^{\prime}\right)=1, v^{\prime} \in \mathbb{R}^{+} .
\end{gathered}
$$

Equations (36)-(39) define initial value problems satisfied by $\tilde{D}_{0}$ and by $D_{0}$, respectively. Recall that the relation between $D_{0}$ and $\tilde{D}_{0}$ is given in (33). It is easy to see that $D_{0}\left(s, v^{\prime}\right)=1, s, v^{\prime} \in \mathbb{R}^{+}$, is a solution of (38), (39). Let us obtain this solution using a procedure that later will be extended to deduce explicit formulae for the functions $D_{j}$ when $j>0$.

Defining $L_{0}, v$ and $\tilde{L}_{0}$ through the relations: $D_{0}\left(s, v^{\prime}\right)=\sqrt{v^{\prime} L_{0}}\left(s, v^{\prime}\right), \quad s, v^{\prime} \in \mathbb{R}^{+}, \quad v=\ln \left(v^{\prime}\right)$, $v^{\prime} \in \mathbb{R}^{+}$(i.e. $\left.v^{\prime}(v)=\mathrm{e}^{v}, \quad v \in \mathbb{R}\right)$ and $\tilde{L}_{0}(s, v)=L_{0}\left(s, v^{\prime}(v)\right), \quad s \in \mathbb{R}^{+}, v \in \mathbb{R}$, problem (38), (39) can be rewritten as follows:

$$
\begin{gathered}
\frac{\partial \tilde{L}_{0}}{\partial s}=\frac{\varepsilon^{2}}{2} \frac{\partial^{2} \tilde{L}_{0}}{\partial v^{2}}-\frac{\varepsilon^{2}}{8} \tilde{L}_{0}, s \in \mathbb{R}^{+}, v \in \mathbb{R}, \\
\tilde{L}_{0}(0, v)=\mathrm{e}^{-v / 2}, v \in \mathbb{R} .
\end{gathered}
$$

The solution of (40), (41) is given by:

$$
\tilde{L}_{0}(s, v)=\int_{-\infty}^{+\infty} \mathrm{d} v^{\prime} \Psi_{0}\left(s, v-v^{\prime}\right) \mathrm{e}^{-v^{\prime} / 2}, s \in \mathbb{R}^{+}, v \in \mathbb{R},
$$

where

$$
\Psi_{0}(s, v)=\frac{1}{\sqrt{2 \pi \varepsilon^{2} s}} \mathrm{e}^{-\varepsilon^{2} s / 8} \mathrm{e}^{-v^{2} /\left(2 \varepsilon^{2} s\right)}, s \in \mathbb{R}^{+}, v \in \mathbb{R} .
$$

The integral (42) when $q=(2 j-1) / 2$ and $j=0$ can be computed using the formula

$$
\begin{aligned}
& \int_{-\infty}^{+\infty} \mathrm{d} v^{\prime} \Psi_{0}\left(s, v-v^{\prime}\right) \mathrm{e}^{q v^{\prime}} \\
& =\mathrm{e}^{-\varepsilon^{2} s / 8} \mathrm{e}^{q v} \mathrm{e}^{s \varepsilon^{2} q^{2} / 2}=\mathrm{e}^{\left(4 q^{2}-1\right) s / 8} \mathrm{e}^{q v}, \\
& s \in \mathbb{R}^{+}, v \in \mathbb{R}, q \in \mathbb{R} .
\end{aligned}
$$

It follows that the solution $\tilde{L}_{0}$ of problem (40), (41) 
is given by:

$$
\tilde{L}_{0}(s, v)=\mathrm{e}^{-v / 2} \mathrm{e}^{s \varepsilon^{2} / 8} \mathrm{e}^{-\varepsilon^{2} s / 8}=\mathrm{e}^{-v / 2}, s \in \mathbb{R}^{+}, v \in \mathbb{R} .
$$

From (45) it follows that

$$
\begin{aligned}
D_{0}\left(s, v^{\prime}\right) & =\sqrt{v^{\prime}} \tilde{L}_{0}\left(s, \ln \left(v^{\prime}\right)\right) \\
& =\sqrt{v^{\prime}} \mathrm{e}^{-\ln \left(v^{\prime}\right) / 2}=1, s, v^{\prime} \in \mathbb{R}^{+} .
\end{aligned}
$$

Let us derive the initial value problems that are satisfied by the functions $D_{j}\left(s, v^{\prime}\right)=\left.\tilde{D}_{j}\left(s, k, v^{\prime}\right)\right|_{k=0}$, $s, v^{\prime} \in \mathbb{R}^{+}, \quad j=1,2, \cdots$. Differentiating $j$ times with respect to $k$ problem (34), (35) and substituting $k=0$ in the resulting equations, we have that when $j=1$ the function $D_{1}\left(s, v^{\prime}\right), s, v^{\prime} \in \mathbb{R}^{+}$, satisfies the following initial value problem:

$$
\begin{gathered}
\frac{\partial D_{1}}{\partial s}=\frac{\varepsilon^{2}}{2} v^{\prime 2} \frac{\partial^{2} D_{1}}{\partial v^{\prime 2}}+\frac{\imath}{2} v^{\prime 2} D_{0}, s, v^{\prime} \in \mathbb{R}^{+}, \\
D_{1}\left(0, v^{\prime}\right)=0, v^{\prime} \in \mathbb{R}^{+},
\end{gathered}
$$

and when $j=2,3, \cdots$ the function $D_{j}\left(s, v^{\prime}\right)$, $s, v^{\prime} \in \mathbb{R}^{+}$, satisfies the following initial value problem:

$$
\begin{aligned}
& \frac{\partial D_{j}}{\partial s}=\frac{\varepsilon^{2}}{2} v^{\prime 2} \frac{\partial^{2} D_{j}}{\partial v^{\prime 2}}-\frac{j}{2}(j-1)\left(v^{\prime}\right)^{2} D_{j-2} \\
&-j \imath \rho \varepsilon v^{\prime 2} \frac{\partial D_{j-1}}{\partial v^{\prime}}+j \frac{\imath}{2} v^{\prime 2} D_{j-1}, \\
& s \in \mathbb{R}^{+}, v^{\prime} \in \mathbb{R}^{+}, j=2,3, \cdots, \\
& D_{j}\left(0, v^{\prime}\right)=0, v^{\prime} \in \mathbb{R}^{+}, j=2,3, \cdots
\end{aligned}
$$

Let us assume that

$$
D_{j}\left(s, v^{\prime}\right)=\sqrt{v^{\prime}} L_{j}\left(s, v^{\prime}\right), s, v^{\prime} \in \mathbb{R}^{+}, j=1,2, \cdots,
$$

and considering the change of variable $v=\ln \left(v^{\prime}\right)$, $v^{\prime} \in \mathbb{R}^{+}$, define the following functions

$\tilde{L}_{j}(s, v)=L_{j}\left(s, v^{\prime}(v)\right)=L_{j}\left(s, \mathrm{e}^{v}\right), \quad s \in \mathbb{R}^{+}, \quad v \in \mathbb{R}$, $j=1,2, \cdots$.

It is easy to see that (47), (48) imply that the function $\tilde{L}_{1}$, is the solution of the following initial value problem:

$$
\begin{gathered}
\frac{\partial \tilde{L}_{1}}{\partial s}=\frac{\varepsilon^{2}}{2} \frac{\partial^{2} \tilde{L}_{1}}{\partial v^{2}}-\frac{\varepsilon^{2}}{8} \tilde{L}_{1}-\imath \rho \varepsilon \mathrm{e}^{v / 2} \frac{\partial \bar{D}_{0}}{\partial v} \\
+\frac{1}{2} \imath \mathrm{e}^{3 v / 2} \bar{D}_{0}, \quad s \in \mathbb{R}^{+}, v \in \mathbb{R}^{+}, \\
\tilde{L}_{1}(0, v)=0, v \in \mathbb{R},
\end{gathered}
$$

where $\quad \bar{D}_{0}(s, v)=D_{0}\left(s, \mathrm{e}^{v}\right)=1, \quad s \in \mathbb{R}^{+}, \quad v \in \mathbb{R}$. Moreover, from (49), (50) it follows that the functions $\tilde{L}_{j}, \quad j=2,3, \cdots$, satisfy the initial value problems:

$$
\begin{gathered}
\frac{\partial \tilde{L}_{j}}{\partial s}=\frac{\varepsilon^{2}}{2} \frac{\partial^{2} \tilde{L}_{j}}{\partial v^{2}}-\frac{\varepsilon^{2}}{8} \tilde{L}_{j}-\frac{j}{2}(j-1) \mathrm{e}^{3 v / 2} \bar{D}_{j-2} \\
-j \imath \rho \varepsilon \mathrm{e}^{v / 2} \frac{\partial \bar{D}_{j-1}}{\partial v}+\frac{j}{2} \imath \mathrm{e}^{3 v / 2} \bar{D}_{j-1}, \\
s \in \mathbb{R}^{+}, v \in \mathbb{R}^{+}, j=2,3, \cdots, \\
\tilde{L}_{j}(0, v)=0, v \in \mathbb{R}, j=2,3, \cdots,
\end{gathered}
$$

where $\quad \bar{D}_{j}(s, v)=D_{j}\left(s, \mathrm{e}^{v}\right), \quad s \in \mathbb{R}^{+}, \quad v \in \mathbb{R}$, $j=2,3, \cdots$.

The solution of (52)-(55) can be written as follows:

$$
\begin{aligned}
\tilde{L}_{j}(s, v)= & \int_{0}^{s} \mathrm{~d} \tau \int_{-\infty}^{+\infty} \mathrm{d} \tilde{v} \Psi_{0}(s-\tau, v-\tilde{v}) \\
& \times\left\{-\frac{j}{2}(j-1) \mathrm{e}^{3 \tilde{v} / 2} \bar{D}_{j-2}(\tau, \tilde{v})-j(\imath \rho \varepsilon) \mathrm{e}^{\tilde{v} / 2} \frac{\partial}{\partial \tilde{v}} \bar{D}_{j-1}(\tau, \tilde{v})+\frac{j}{2} \imath \mathrm{e}^{3 \tilde{v} / 2} \bar{D}_{j-1}(\tau, \tilde{v})\right\}, \\
& s \in \mathbb{R}^{+}, v \in \mathbb{R}, j=1,2, \cdots,
\end{aligned}
$$

where we have defined $\bar{D}_{-1}(s, v)=0, s \in \mathbb{R}^{+}, \quad v \in \mathbb{R}$.

Let us give the explicit expressions of

$D_{j}\left(s, v^{\prime}\right)=\sqrt{v^{\prime}} \tilde{L}_{j}\left(s, \ln \left(v^{\prime}\right)\right), s, v^{\prime} \in \mathbb{R}^{+}$, and of

$\mathcal{L}_{j}^{*}\left(t, \tilde{\xi}_{0}, \tilde{v}_{0}\right), \quad t \in \mathbb{R}^{+}$, when $j=1,2,3,4$. Recall that $D_{j}\left(s, v^{\prime}\right)=\sqrt{v^{\prime}} \tilde{L}_{j}\left(s, \ln \left(v^{\prime}\right)\right), s, v^{\prime} \in \mathbb{R}^{+}, \quad j=1,2, \cdots, \quad$ and that $D_{0}\left(s, v^{\prime}\right)=1, s, v^{\prime} \in \mathbb{R}^{+}$. Using Formulae (44), (56) we have:

$$
\imath D_{1}\left(s, v^{\prime}\right)=-\frac{1}{2}\left(\frac{v^{\prime}}{\varepsilon}\right)^{2} \mathrm{e}^{\varepsilon^{2} s}\left(1-\mathrm{e}^{-\varepsilon^{2} s}\right), \quad s, v^{\prime} \in \mathbb{R}^{+},
$$




$$
\begin{aligned}
& \imath D_{3}\left(s, v^{\prime}\right)=-6 \rho\left(\frac{v^{\prime}}{\varepsilon}\right)^{3} \mathrm{e}^{3 \varepsilon^{2} s} \times\left[\left(\frac{1-\mathrm{e}^{-2 \varepsilon^{2} s}}{2}\right)-\left(\frac{1-\mathrm{e}^{-3 \varepsilon^{2} s}}{3}\right)\right]+18 \rho^{2}\left(\frac{v^{\prime}}{\varepsilon}\right)^{4} \mathrm{e}^{6 \varepsilon^{2} s}\left[\left(\frac{1-\mathrm{e}^{-3 \varepsilon^{2} s}}{18}\right)-\left(\frac{1-\mathrm{e}^{-5 \varepsilon^{2} s}}{10}\right)+\left(\frac{1-\mathrm{e}^{-6 \varepsilon^{2} s}}{18}\right)\right] \\
& +3\left(\frac{v^{\prime}}{\varepsilon}\right)^{4} \mathrm{e}^{6 \varepsilon^{2} s}\left[\left(\frac{1-\mathrm{e}^{-5 \varepsilon^{2} s}}{5}\right)-\left(\frac{1-\mathrm{e}^{-6 \varepsilon^{2} s}}{6}\right)\right]-3 \rho\left(\frac{v^{\prime}}{\varepsilon}\right)^{5} \mathrm{e}^{10 \varepsilon^{2} s}\left[\left(\frac{1-\mathrm{e}^{-4 \varepsilon^{2} s}}{60}\right)+\left(\frac{1-\mathrm{e}^{-7 \varepsilon^{2} s}}{42}\right)-\left(\frac{1-\mathrm{e}^{-9 \varepsilon^{2} s}}{10}\right)+\left(\frac{1-\mathrm{e}^{-10 \varepsilon^{2} s}}{15}\right)\right] \\
& +\frac{1}{4}\left(\frac{v^{\prime}}{\varepsilon}\right)^{6} \mathrm{e}^{15 \varepsilon^{2} s}\left[\left(\frac{1-\mathrm{e}^{-9 \varepsilon^{2} s}}{90}\right)-3\left(\frac{1-\mathrm{e}^{-14 \varepsilon^{2} s}}{70}\right)+\left(\frac{1-\mathrm{e}^{-15 \varepsilon^{2} s}}{30}\right)\right], s, v^{\prime} \in \mathbb{R}^{+},
\end{aligned}
$$

and finally

where $I_{1}, I_{2}$ and $I_{3}$ are the following functions:

$$
\begin{aligned}
& \imath D_{4}\left(s, v^{\prime}\right)=I_{1}\left(s, v^{\prime}\right)+I_{2}\left(s, v^{\prime}\right)+I_{3}\left(s, v^{\prime}\right), \quad s, v^{\prime} \in \mathbb{R}^{+},(60) \\
& I_{1}\left(s, v^{\prime}\right)=4 \rho^{2}\left(\frac{v^{\prime}}{\varepsilon}\right)^{4} \mathrm{e}^{6 \varepsilon^{2} s} \times\left[\left(1-\mathrm{e}^{-3 \varepsilon^{2} s}\right)-9\left(\frac{1-\mathrm{e}^{-5 \varepsilon^{2} s}}{5}\right)+\left(1-\mathrm{e}^{-6 \varepsilon^{2} s}\right)\right] \\
& -4 \rho^{3}\left(\frac{v^{\prime}}{\varepsilon}\right)^{5} \mathrm{e}^{10 \varepsilon^{2} s}\left[\left(\frac{1-\mathrm{e}^{-4 \varepsilon^{2} s}}{5}\right)-4\left(\frac{1-\mathrm{e}^{-7 \varepsilon^{2} s}}{7}\right)+4\left(\frac{1-\mathrm{e}^{-9 \varepsilon^{2} s}}{5}\right)-2\left(\frac{1-\mathrm{e}^{-10 \varepsilon^{2} s}}{5}\right)\right] \\
& -4 \rho\left(\frac{v^{\prime}}{\varepsilon}\right)^{5} \mathrm{e}^{10 \varepsilon^{2} s}\left[\left(\frac{1-\mathrm{e}^{-4 \varepsilon^{2} s}}{10}\right)-4\left(\frac{1-\mathrm{e}^{-9 \varepsilon^{2} s}}{15}\right)+\left(\frac{1-\mathrm{e}^{-10 \varepsilon^{2} s}}{5}\right)\right] \\
& +2 \rho^{2}\left(\frac{v^{\prime}}{\varepsilon}\right)^{6} \mathrm{e}^{15 \varepsilon^{2} s}\left[3\left(\frac{1-\mathrm{e}^{-5 \varepsilon^{2} s}}{70}\right)-\left(\frac{1-\mathrm{e}^{-9 \varepsilon^{2} s}}{18}\right)-5\left(\frac{1-\mathrm{e}^{-12 \varepsilon^{2} s}}{84}\right)+3\left(\frac{1-\mathrm{e}^{-14 \varepsilon^{2} s}}{14}\right)-2\left(\frac{1-\mathrm{e}^{-15 \varepsilon^{2} s}}{15}\right)\right] \\
& -\rho\left(\frac{v^{\prime}}{\varepsilon}\right)^{7} \mathrm{e}^{21 \varepsilon^{2} s}\left[\left(\frac{1-\mathrm{e}^{-6 \varepsilon^{2} s}}{630}\right)-\left(\frac{1-\mathrm{e}^{-15 \varepsilon^{2} s}}{225}\right)+9\left(\frac{1-\mathrm{e}^{-20 \varepsilon^{2} s}}{700}\right)-\left(\frac{1-\mathrm{e}^{-21 \varepsilon^{2} s}}{105}\right)\right], s, v^{\prime} \in \mathbb{R}^{+}, \\
& I_{2}\left(s, v^{\prime}\right)=+6\left(\frac{v^{\prime}}{\varepsilon}\right)^{4} \mathrm{e}^{6 \varepsilon^{2} s} \times\left[\left(\frac{1-\mathrm{e}^{-5 \varepsilon^{2} s}}{5}\right)-\left(\frac{1-\mathrm{e}^{-6 \varepsilon^{2} s}}{6}\right)\right]-6 \rho\left(\frac{v^{\prime}}{\varepsilon}\right)^{5} \mathrm{e}^{10 \varepsilon^{2} s} \times\left[\left(\frac{1-\mathrm{e}^{-7 \varepsilon^{2} s}}{21}\right)-\left(\frac{1-\mathrm{e}^{-9 \varepsilon^{2} s}}{9}\right)+\left(\frac{1-\mathrm{e}^{-10 \varepsilon^{2} s}}{15}\right)\right] \\
& -\frac{3}{10}\left(\frac{v^{\prime}}{\varepsilon}\right)^{6} \mathrm{e}^{15 \varepsilon^{2} s} \times\left[\left(\frac{1-\mathrm{e}^{-9 \varepsilon^{2} s}}{27}\right)-\left(\frac{1-\mathrm{e}^{-14 \varepsilon^{2} s}}{7}\right)+\left(\frac{1-\mathrm{e}^{-15 \varepsilon^{2} s}}{9}\right)\right], s, v^{\prime} \in \mathbb{R}^{+},
\end{aligned}
$$

and

$$
\begin{aligned}
I_{3}\left(s, v^{\prime}\right)= & -12 \rho\left(\frac{v^{\prime}}{\varepsilon}\right)^{5} \mathrm{e}^{10 \varepsilon^{2} s} \times\left[\left(\frac{1-\mathrm{e}^{-7 \varepsilon^{2} s}}{35}\right)-\left(\frac{1-\mathrm{e}^{-9 \varepsilon^{2} s}}{18}\right)+\left(\frac{1-\mathrm{e}^{-10 \varepsilon^{2} s}}{30}\right)\right] \\
& +2 \rho^{2}\left(\frac{v^{\prime}}{\varepsilon}\right)^{6} \mathrm{e}^{15 \varepsilon^{2} s} \times\left[\left(\frac{1-\mathrm{e}^{-9 \varepsilon^{2} s}}{45}\right)-\left(\frac{1-\mathrm{e}^{-12 \varepsilon^{2} s}}{12}\right)+9\left(\frac{1-\mathrm{e}^{-14 \varepsilon^{2} s}}{70}\right)-\left(\frac{1-\mathrm{e}^{-15 \varepsilon^{2} s}}{15}\right)\right] \\
& +6\left(\frac{v^{\prime}}{\varepsilon}\right)^{6} \mathrm{e}^{15 \varepsilon^{2} s}\left[\left(\frac{1-\mathrm{e}^{-9 \varepsilon^{2} s}}{270}\right)-\left(\frac{1-\mathrm{e}^{-14 \varepsilon^{2} s}}{70}\right)+\left(\frac{1-\mathrm{e}^{-15 \varepsilon^{2} s}}{90}\right)\right] \\
& -\rho\left(\frac{v^{\prime}}{\varepsilon}\right)^{7} \mathrm{e}^{21 \varepsilon^{2} s}\left[3\left(\frac{1-\mathrm{e}^{-11 \varepsilon^{2} s}}{770}\right)-\left(\frac{1-\mathrm{e}^{-15 \varepsilon^{2} s}}{150}\right)-\left(\frac{1-\mathrm{e}^{-18 \varepsilon^{2} s}}{126}\right)+3\left(\frac{1-\mathrm{e}^{-20 \varepsilon^{2} s}}{100}\right)-2\left(\frac{1-\mathrm{e}^{-21 \varepsilon^{2} s}}{105}\right)\right] \\
& -\frac{1}{20}\left(\frac{v^{\prime}}{\varepsilon}\right)^{8} \mathrm{e}^{28 \varepsilon^{2} s}\left[\left(\frac{1-\mathrm{e}^{-13 \varepsilon^{2} s}}{819}\right)-\left(\frac{1-\mathrm{e}^{-22 \varepsilon^{2} s}}{198}\right)+\left(\frac{1-\mathrm{e}^{-27 \varepsilon^{2} s}}{63}\right)-\left(\frac{1-\mathrm{e}^{-28 \varepsilon^{2} s}}{84}\right)\right], s, v^{\prime} \in \mathbb{R}^{+} .
\end{aligned}
$$


Recall that $s=t-t^{\prime}$. From (30), (46), (57)-(60), choosing $t^{\prime}=0, \xi^{\prime}=\tilde{\xi}_{0}, v^{\prime}=\tilde{v}_{0}$ we have that $s=t$ and the first five moments with respect to zero of $\xi_{t}$, $t \in \mathbb{R}^{+}$, are given by:

$$
\begin{gathered}
\mathcal{L}_{0}^{*}\left(t, \tilde{\xi}_{0}, \tilde{v}_{0}\right)=D_{0}\left(t, \tilde{v}_{0}\right)=1, t \in \mathbb{R}^{+}, \tilde{\xi}_{0} \in \mathbb{R}, \tilde{v}_{0} \in \mathbb{R}^{+}, \\
\mathcal{L}_{1}^{*}\left(t, \tilde{\xi}_{0}, \tilde{v}_{0}\right)=\tilde{\xi}_{0} D_{0}\left(t, \tilde{v}_{0}\right)+\imath D_{1}\left(t, \tilde{v}_{0}\right), \\
t \in \mathbb{R}^{+}, \tilde{\xi}_{0} \in \mathbb{R}, \tilde{v}_{0} \in \mathbb{R}^{+}, \\
\mathcal{L}_{2}^{*}\left(t, \tilde{\xi}_{0}, \tilde{v}_{0}\right)=\tilde{\xi}_{0}^{2} D_{0}\left(t, \tilde{v}_{0}\right)+2 \tilde{\xi}_{0} \imath D_{1}\left(t, \tilde{v}_{0}\right) \\
-3 \tilde{\xi}_{0} D_{1}\left(t, \tilde{v}_{0}\right)-D_{2}\left(t, \tilde{v}_{0}\right), \\
t \in \mathbb{R}^{+}, \tilde{\xi}_{0} \in \mathbb{R}, \tilde{v}_{0} \in \mathbb{R}^{+}, \\
\mathcal{L}_{3}^{*}\left(t, \tilde{\xi}_{0}, \tilde{v}_{0}\right)=\tilde{\xi}_{0}^{3} D_{0}\left(t, \tilde{v}_{0}\right)+3 \tilde{\xi}_{0}^{2} \imath D_{1}\left(t, \tilde{v}_{0}\right) \\
-3 \tilde{\xi}_{0} D_{2}\left(t, \tilde{v}_{0}\right)-\imath D_{3}\left(t, \tilde{v}_{0}\right), \\
t \in \mathbb{R}^{+}, \tilde{\xi}_{0} \in \mathbb{R}, \tilde{v}_{0} \in \mathbb{R}^{+}, \\
\mathcal{L}_{4}^{*}\left(t, \tilde{\xi}_{0}, \tilde{v}_{0}\right)=\tilde{\xi}_{0}^{4} D_{0}\left(t, \tilde{v}_{0}\right)+4 \tilde{\xi}_{0}^{4} \imath D_{1}\left(t, \tilde{v}_{0}\right) \\
-6 \tilde{\xi}_{0}^{2} D_{2}\left(t, \tilde{v}_{0}\right)-4 \tilde{\xi}_{0} \imath D_{3}\left(t, \tilde{v}_{0}\right) \\
+D_{4}\left(t, \tilde{v}_{0}\right), t \in \mathbb{R}^{+}, \tilde{\xi}_{0} \in \mathbb{R}, \tilde{v}_{0} \in \mathbb{R}^{+} .
\end{gathered}
$$

The moments $\mathcal{L}_{1}^{*}, \mathcal{L}_{2}^{*}, \mathcal{L}_{3}^{*}, \mathcal{L}_{4}^{*}$ depend on the parameters of the lognormal SABR model $\varepsilon, \rho, \tilde{v}_{0}$ and on the time $t$. In particular, $L_{1}^{*}$ depends on $\varepsilon, \tilde{v}_{0}$ and $t$, while $\mathcal{L}_{2}^{*}, \mathcal{L}_{3}^{*}, \mathcal{L}_{4}^{*}$ depend on $\varepsilon, \tilde{v}_{0}, \rho$ and $t$. The moment $\mathcal{L}_{0}^{*}$ does not depend on $\varepsilon, \tilde{v}_{0}, \rho, t$ and cannot be used in the study of calibration problems for the lognormal SABR model. Formulaes (65)-(68) for the moments of the lognormal SABR model are the new closed form moment formulae announced in Section 1 and are the formulae used in the next sections to solve the calibration problems discussed previously. Analogous formulae can be deduced (at least in principle) for all the remaining moments $\mathcal{L}_{n, m}$ defined in (27). Of course as $n, m$ increase the formulae for $\mathcal{L}_{n, m}$ become more and more involved. Note that, as already said in Section 1, closed form formulae of observable quantities implicitly defined by (9), (10), (3), (4) such as (65)-(68) are very useful to build computationally efficient methods to solve calibration problems.

In [4], Formulae analogous to (65)-(68) for the moments of the forward prices/rates variable of the normal SABR model (6), (2), (3), (4) are derived and used to solve calibration problems.

\section{Two Calibration Problems for the Lognormal SABR Model}

Let us study the calibration problems of the lognormal SABR model (11)-(14) announced in Section 1. Recall that the parameters $\varepsilon, \rho, \tilde{v}_{0}$ are the unknowns and that we want to determine these parameters starting from the knowledge of a set of data. We consider the sets of data specified previously. The corresponding calibration problems are formulated using the closed form Formulaes (65)-(68) for the moments of the logarithm of the forward prices/rates variable and are solved numerically.

Let us begin formulating the first calibration problem. Let $T>0$ be given. We consider multiple independent trajectories of the lognormal SABR model (11), (12) associated to the initial conditions (13), (14) assigned at time $t=0$. The set of data of the first calibration problem is the set of the logarithms of the forward prices/ rates observed at time $t=T$ in this set of trajectories.

In particular, letting $n$ be a positive integer, we consider $n$ independent copies $\xi_{T}^{i}, i=1,2, \cdots, n$, of the random variable $\xi_{T}$ solution at time $t=T$ of (11)-(14). For $i=1,2, \cdots, n$ let $\hat{\xi}_{T}^{i}$ be a realization of $\xi_{T}^{i}$. The set:

$$
\mathcal{D}_{1}=\left\{\hat{\xi}_{T}^{i}, i=1,2, \cdots, n\right\},
$$

is the data sample used in the following calibration problem:

Calibration problem 1: multiple trajectories calibration problem.

Given $T>0, n>0$ and the data set $\mathcal{D}_{1}$ defined in (69), reconstruct the values of the parameters $\varepsilon, \rho$ and $\tilde{v}_{0}$ of the lognormal SABR model (11)-(14).

To solve this calibration problem we compare the theoretical values of the four moments $\mathcal{L}_{j}^{*}, \quad j=1,2,3,4$, given by (65)-(68) with the estimates of these moments obtained from the sample $\mathcal{D}_{1}$ of the observed data.

It is easy to see that the random variables:

$$
\Lambda_{j}(n, T)=\frac{1}{n} \sum_{i=1}^{n}\left(\xi_{T}^{i}\right)^{j}, j=1,2,3,4,
$$

are unbiased estimators of, respectively, $\mathcal{L}_{j}^{*}$,

$j=1,2,3,4$. For $j=1,2,3,4$ let us consider the realization $\hat{\Lambda}_{j}(n, T)$, in the data sample $\mathcal{D}_{1}$, of the random variable $\Lambda_{j}(n, T)$, that is:

$$
\hat{\Lambda}_{j}(n, T)=\frac{1}{n} \sum_{i=1}^{n}\left(\hat{\xi}_{T}^{i}\right)^{j}, j=1,2,3,4 .
$$

The unknown parameters $\varepsilon, \rho, \tilde{v}_{0}$ of the normal SABR model can be determined as solutions of the following constrained nonlinear least-squares problem:

$$
\min _{\varepsilon, \rho, \tilde{v}_{0}} \sum_{j=1}^{4} \sigma_{j}\left(\mathcal{L}_{j}^{*}\left(T, \tilde{\xi}_{0}, \tilde{v}_{0}\right)-\hat{\Lambda}_{j}(n, T)\right)^{2},
$$

subject to the constraints:

$$
\varepsilon>0,-1<\rho<1, \tilde{v}_{0}>0,
$$

where $\sigma_{j}, j=1,2,3,4$, are non negative weights that will be chosen in Section 4. 
Note that roughly speaking when $n$ increases the "quality" of the moments $\hat{\Lambda}_{j}(n, T), \quad j=1,2,3,4$, estimated from the data sample increases and this should make easier to solve satisfactorily the Calibration problem 1 .

The numerical experience shows that the constrained nonlinear least-squares problem (72), (73) has many local minimizers with similar objective function values. This means that the solution of problem (72), (73) is sensitive to the choice of the initial guess of the minimization procedure used to solve it. To construct good initial guesses of problem (72), (73) we take a closer look to the explicit expression of the moment Formulae (65)-(68). In particular, we consider the asymptotic expansion of the moment $\mathcal{L}_{1}^{*}$ (formula (65)) when $\varepsilon^{2} t \rightarrow 0$. Let $T_{1}, T_{2}$ be such that $0<T_{1}<T_{2}$, we approximate $\mathcal{L}_{1}^{*}$ with the first- and the second-order Taylor's expansions of base point $t=0$. The first-order approximation of $\mathcal{L}_{1}^{*}$ when $t=T_{1}$ is used to obtain the initial guess for the parameter $\tilde{v}_{0}$. The second-order approximation of $\mathcal{L}_{1}^{*}$ when $t=T_{2}$ is used to obtain the initial guess for the parameter $\varepsilon$. To build an initial guess of the parameter $\rho$ it is necessary to use higher-order moments. We prefer to exploit the fact that $-1<\rho<1$ and that the availability of the explicit moment formulae makes computationally very efficient the solution of problem (72), (73). This means that, when necessary, at an affordable computational cost, it is possible to use multiple initial guesses for the parameter $\rho$.

The data sample $\mathcal{D}_{1}$ defined in (69) used in Calibration problem 1 to formulate problem (72), (73) must be completed with the auxiliary data $\hat{\xi}_{T_{1}}^{i}, \hat{\xi}_{T_{2}}^{i}$, $i=1,2, \cdots, n$, (observed at time $t=T_{1}$ and $t=T_{2}$ ) needed to build the initial guess of the minimization procedure used to solve problem (72), (73). For simplicity, it is possible to choose $T=T_{1}$ or $T=T_{2}$ as it is done in the numerical example discussed in Section 4. In this case, the data contained in (69) are used both to formulate the nonlinear least-squares problem (see (72), (73)) and to obtain the initial guess for $\tilde{v}_{0}$ (when $T=T_{1}$ ) or, the initial guess for $\varepsilon$ (when $T=T_{2}$ ).

This set of data is realistic in several contexts of science and engineering where, for example, the observations are obtained in experiments done in a laboratory. In fact, repeated experiments are a routine work in a laboratory. However, most of the times this is not realistic for observations made in the financial markets where usually it is not possible to repeat the "experiment". That is, in the financial markets repeated observations at a given time $t>0$ of independent realizations of the forward prices/rates random variable $\xi_{\text {t }}$ are usually not available. This is a serious concern which implies that the Calibration problem 1 is of limited in- terest in finance.

The second calibration problem for the lognormal SABR model (11)-(14) overcomes this difficulty. In fact, the data sample considered in the second calibration problem is the set of the logarithms of the forward prices/rates observed on a discrete set of known time values along a single trajectory of the lognormal SABR model. This data sample is easily available in the financial markets. It can be identified with a time series of the logarithms of the forward prices/rates observed in the financial market.

Going into details, let $M$ be a positive integer and let $t_{0}, t_{1}, \cdots, t_{M}$ be $M+1$ discrete time values such that $t_{i}>t_{i-1}, \quad i=1,2, \cdots, M$, and $t_{0}=0$. Recall that the times $t_{i}, i=0,1, \cdots, M$, are known. The data of the second calibration problem are the logarithms of the forward prices/rates observed at the times $t_{0}, t_{1}, \cdots, t_{M}$. For $i=1,2, \cdots, M$ let us denote by $\hat{\xi}_{i}$ the logarithm of the forward prices/rates observed at time $t=t_{i}$ along one trajectory of the stochastic process $\xi_{t}, t>0$. The set:

$$
\mathcal{D}_{2}=\left\{\hat{\xi}_{i}, i=1,2, \cdots, M\right\},
$$

is the data sample used in the following calibration problem:

Calibration problem 2: single trajectory calibration problem.

Given $M>0, M+1$ discrete time values $t_{0}, t_{1}, \cdots, t_{M}$, such that $t_{i}>t_{i-1}, \quad i=1,2, \cdots, M$, and $t_{0}=0$, and given the data set $\mathcal{D}_{2}$ defined in (74), determine the values of the parameters $\varepsilon, \rho$ and $\tilde{v}_{0}$ of the lognormal SABR model (11)-(14).

The Calibration problem 2 can be formulated as the following constrained nonlinear least-squares problem:

$$
\min _{\varepsilon, \rho, \tilde{v}_{0}} \sum_{i=1}^{M} \sum_{j=1}^{4} \omega_{i, j}\left(\mathcal{L}_{j}^{*}\left(t_{i}, \tilde{\xi}_{0}, \tilde{v}_{0}\right)-\left(\hat{\xi}_{i}\right)^{j}\right)^{2},
$$

subject to the constraints (73). The constants $\omega_{i, j}$, $i=1,2, \cdots, M, \quad j=1,2,3,4$, in (75) are non negative weights that will be chosen in Section 4. Note that when $M$ increases the "quality" of the terms $\left(\hat{\xi}_{i}\right)^{j}$, $i=1,2, \cdots, M, \quad j=1,2,3,4$, does not increase, it is only the number of addenda of (75) that increases. For this reason we expect Calibration problem 2 to be more difficult than Calibration problem 1.

The numerical experience with problem (75), (73) shows that the behaviour of the constrained nonlinear least-squares problem (75), (73) is similar to the behaviour of problem (72), (73). This implies that the availability of a good initial guess for the numerical optimization algorithm used to solve (75), (73) is very helpful to obtain a satisfactory solution. In order to build this initial guess we exploit the moment formulae derived in Section 2. In particular, we use the first- and the 
second-order Taylor's approximations of $\mathcal{L}_{1}^{*}$ (see (65)) with base point $t=0$. From the first-order Taylor's approximation at $t=0$ of $\mathcal{L}_{1}^{*}$ evaluated at the beginning of the trajectory (i.e. using the observations $\hat{\xi}_{i}$ with $i$ small, that is $i=1,2, \cdots, 10$ in the numerical example of Section 4) we obtain the initial guess of $\tilde{v}_{0}$. From the second-order Taylor's approximation at $t=0$ of $\mathcal{L}_{1}^{*}$ evaluated at the end of the trajectory (i.e. using the observations $\hat{\xi}_{i}$ with $i$ close to $M$, that is $i=91,92, \cdots, 100$ in the numerical example of Section 4) we obtain the initial guess of $\varepsilon$. Sometimes also the first-order Taylor's approximation of $\frac{\mathrm{d} \mathcal{L}_{1}^{*}}{\mathrm{~d} t}$ with base point $t=0$ is used to construct the initial guess of the numerical optimization algorithm used to solve (75), (73). In this last case the first-order Taylor's approximation at $t=0$ of $\mathcal{L}_{1}^{*}$ is used to obtain the initial guess of the parameter $\tilde{v}_{0}$ and the first-order Taylor's approximation at $t=0$ of $\frac{\mathrm{d} \mathcal{L}_{1}^{*}}{\mathrm{~d} t}$ is used to obtain the initial guess of the parameter $\varepsilon$. These approximations are evaluated at the beginning of the trajectory. As explained more in detail in Section 4, in financial applications a priori information about $\rho$ is available. That is, due to the financial meaning of the variables, we must expect $\rho<0$. In Section 4 we exploit this information to choose an initial guess for the parameter $\rho$.

\section{Some Numerical Experiments}

In this section we discuss three numerical experiments. In the first numerical experiment we solve the Calibration problem 1 using synthetic data. In the second and third numerical experiments we solve the Calibration problem 2 using, respectively, synthetic and real data. The real data studied are the data belonging to a time series of exchange rates between currencies (euro/U.S. dollar exchange rates).

The numerical experiments presented in this section can be "interpreted" as follows. As already said, the first numerical experiment can be seen as a "physical experiment" done in the context of a scientific laboratory where it is possible to make repeated observations of the same quantity. This type of experiment usually is based on a "physical model" (i.e. in this case the lognormal SABR model) where the parameters of the model have a precise physical meaning (i.e. they are masses, charges, ...). In these circumstances the main scope of a calibration problem (such as Calibration problem 1) is to determine the numerical values of these parameters in the best possible way. Note that in this kind of experiments usually a more accurate value of the parameters can be obtained increasing the numerousness of the data sample used in the calibration problem. The second and third numerical experiments can be seen as experiments in finance or in a different context where it is not possible to make repeated observations of the same quantity. Note that in mathematical finance the model and its parameters are mainly an auxiliary tool. In fact, the model is simply an instrument to interpret the data or to forecast future data. In the practice of the financial markets the calibrated financial models (such as the calibrated lognormal SABR model) are used to do option's pricing and to hedge portfolios. In these contexts it is not really important to know the exact values of the model parameters. In some sense even the existence of "exact" values for the model parameters can be debated. In mathematical finance the key fact is to show that the calibrated model is able to interpret the observations, that is, for example, to show the consistency of the option prices computed using the calibrated model with the option prices observed in the market.

Let us describe the setting of the first numerical experiment. Let $T>0$ be given and $n, m$ be positive integers. Let $\Delta t=T / m$ be the time increment and $t_{i}=i \Delta t, \quad i=0,1, \cdots, m$, be a discrete set of equispaced time values. Let $\xi_{t_{m}}=\xi_{T}, v_{t_{m}}=v_{T}$ be the solutions of (11)-(14) at time $t=T$. The $n$ independent realizations $\hat{\xi}_{T}^{i}, i=1,2, \cdots, n$, of the random variable $\xi_{T}$ used as data in Calibration problem 1 are approximated integrating numerically $n$ times (in correspondence of different realizations of the Wiener processes) the lognormal SABR model (11)-(14) in the time interval $[0, T]$ using the explicit Euler method (see [14]).

In the numerical example we choose $T=1, m=100$, $n=1000, \quad \varepsilon=0.1, \quad \rho=-0.2 \quad, \quad \xi_{0}=\tilde{\xi}_{0}=1 \quad$ and $v_{0}=\tilde{v}_{0}=0.5$. The parameters

$$
\left(\varepsilon, \rho, \tilde{v}_{0}\right)=(0.1,-0.2,0.5)
$$

are the "true" values of the unknowns of the calibration problem considered (i.e. they are the values of the unknowns used to generate the data). We reconstruct these unknown parameters solving Calibration problem 1 using as data sample the set of the logarithms of the forward prices/rates observed at time $t=T=1$ in $n=1000$ independent trajectories of the lognormal SABR model (11)-(14) (with the parameter values given in (76) and $\left.\xi_{0}=\tilde{\xi}_{0}=1\right)$. These trajectories are approximated integrating numerically using the explicit Euler method the model (11)-(14). In particular, when $n=1000$ let us denote by $\hat{\xi}_{T=1}^{i}, \quad i=1,2, \cdots, n$, the approximations of $\hat{\xi}_{T=1}^{i}, i=1,2, \cdots, n$, obtained at time $t=T=1$ integrating with the explicit Euler method $n=1000$ independent trajectories of the model (11)-(14) (with the parameter values given in (76) and $\left.\xi_{0}=\tilde{\xi}_{0}=1\right)$. The set: 


$$
\hat{\mathcal{D}}_{T=1, n=1000}=\left\{\hat{\hat{\xi}}_{T=1}^{i}, i=1,2, \cdots, 1000\right\},
$$

is the sample of synthetic data used to solve Calibration problem 1.

In a similar way when we choose $T=100, n=1000$ (leaving the other parameters unchanged) we generate the data set $\hat{\mathcal{D}}_{T=100, n=1000}=\left\{\hat{\hat{\xi}}_{T=100}^{i}, i=1,2, \cdots, 1000\right\}$. As explained in Section 3, this second data sample is used in the construction of the initial guess of the numerical optimization algorithm used to solve the constrained nonlinear least-squares problem (72), (73) corresponding to the data sample $\hat{\mathcal{D}}_{T=1, n=1000}$.

The data sets $\hat{\mathcal{D}}_{T=1, n=1000}$ and $\hat{\mathcal{D}}_{T=100, n=1000}$ used in the numerical experiment are available at [15].

Using $\hat{\mathcal{D}}_{T=1, n=1000}$ and $\hat{\mathcal{D}}_{T=100, n=1000}$ and the first- and second-order Taylor's approximations of $\mathcal{L}_{1}^{*}$ with base point $t=0$, as suggested in Section 3 , we find $\tilde{v}_{0}^{i n}=0.515$ and $\varepsilon^{i n}=0.099$ as initial guesses of, respectively, $\tilde{v}_{0}$ and $\varepsilon$. Note that in the notation of Section 3 we have chosen $T=T_{1}=1$ and $T_{2}=100$. The initial guess $\rho^{\text {in }}$ of $\rho$ is chosen as $\rho^{\text {in }}=-0.05$.

Given $\left(\varepsilon^{i n}, \rho^{i n}, \tilde{v}_{0}^{i n}\right)=(0.099,-0.05,0.515)$ as initial guess, the nonlinear least-squares problem (72), (73) is solved using $\hat{\mathcal{D}}_{T=1, n=1000}$ as data sample. In this numerical example the moments considered in (72) are all of the same order of magnitude so that it is possible to choose in (72) the weights $\sigma_{j}=1, j=1,2,3,4$. Note that Formulae (65)-(68) suggest that in general the weight $\sigma_{j}$ must decrease when the index $j$ increases.

The nonlinear least-squares problem (72), (73) is solved using the FMINCON routine of Matlab. The solution found starting from the initial guess

$$
\begin{aligned}
& \left(\varepsilon^{i n}, \rho^{i n}, \tilde{v}_{0}^{i n}\right)=(0.099,-0.05,0.515) \text { is: } \\
& \quad\left(\varepsilon, \rho, \tilde{v}_{0}\right)=\left(\varepsilon^{*}, \rho^{*}, \tilde{v}_{0}^{*}\right)=(0.076,-0.222,0.508) .
\end{aligned}
$$

The relative $L^{2}$-error of the initial guess $\left(\varepsilon^{\text {in }}, \rho^{\text {in }}, \tilde{v}_{0}^{\text {in }}\right)=(0.099,-0.05,0.515)$ with respect to the "true" solution (76) is 0.275 . The relative $L^{2}$-error of the solution (78) of the least-squares problem with respect to the "true" solution (76) is 0.062 .

To study the sensitivity of the solution procedure proposed with respect to the presence of noise in the data we add noise of known statistical properties to the synthetic data contained in $\hat{\mathcal{D}}_{T=1, n=1000}, \hat{\mathcal{D}}_{T=100, n=1000}$ and we study the quality of the solutions of Calibration problem 1 found as a function of the noise properties. In particular given $\vartheta_{1}>0$ let us consider the following "noisy" data sample:

$$
\hat{\mathcal{E}}_{T=1, n=1000}=\left\{\hat{\hat{\xi}}_{T=1}^{i}\left(1+\vartheta_{1}(1-2 \text { rand })\right), i=1,2, \cdots, 1000\right\},
$$

where rand is a random entry taken from a uniform distribution on the interval $(0,1)$. In a similar way we add noise to $\hat{\mathcal{D}}_{T=100, n=1000}$ to obtain the "noisy" data sample $\hat{\mathcal{E}}_{T=100, n=1000}$.

Given $\vartheta_{1}>0$ we compute the relative $L^{2}$-error between the "true" solution (76) and the solution of Calibration problem 1 found with the numerical procedure described above. We repeat the entire procedure $N_{1}$ times and we compute the mean of the $L^{2}$-relative errors of the $N_{1}$ solutions found. We denote by $\bar{E}_{\vartheta_{1}, N_{1}, n}$ this mean relative error. The mean relative errors $\bar{E}_{\vartheta_{1}, N_{1}=1000, n=1000}$ obtained when $N_{1}=1000$, $n=1000$ and $\vartheta_{1}=0.01,0.05,0.1$ are shown in Table 1 .

As already explained, it is expected that in this experiment a more accurate value of the parameters can be obtained by increasing the amount of the data sample used in the calibration problem. To validate this idea, we increase the number $n$ of the data samples involved in the experiment. We consider $n=10000$ and we construct the data samples $\hat{\mathcal{D}}_{T=1, n=10000}, \hat{\mathcal{D}}_{T=100, n=10000}$ and the corresponding "noisy" data samples $\hat{\mathcal{E}}_{T=1, n=10000}$, $\hat{\mathcal{E}}_{T=100, n=10000}$. The data sets $\hat{\mathcal{D}}_{T=1, n=10000}$ and $\hat{\mathcal{D}}_{T=100, n=10000}^{T=1, n=10000}$, used in the numerical experiment are available at [15]. Given $\vartheta_{1}>0$ we compute the mean relative errors $\bar{E}_{\vartheta_{1}, N_{1}=1000, n=10000}$ obtained when $N_{1}=1000, n=10000$ and $\vartheta_{1}=0.01,0.05,0.1$. The results obtained are shown in Table 2. The comparison of Tables 1 and $\mathbf{2}$ shows a substantial reduction of the mean relative errors when $\vartheta_{1}=0.05$ and $\vartheta_{1}=0.1$ and only a marginal reduction of the mean relative errors when $\vartheta_{1}=0.01$. This suggests that the presence of noise even in small quantity degrades the solution obtained.

The second numerical experiment presented consists in solving Calibration problem 2 using a sample of synthetic data. Given the number of observations $M>0$

Table 1. Calibration problem 1: the mean relative error $\bar{E}_{\vartheta_{1}, N_{1}=1000, n=1000}$ as a function of $\vartheta_{1}$.

\begin{tabular}{cc}
\hline$\vartheta_{1}$ & $\bar{E}_{\vartheta_{1}, N_{1}=100, n=1000}$ \\
\hline 0.01 & 0.124 \\
0.05 & 0.215 \\
0.1 & 0.328 \\
\hline
\end{tabular}

Table 2. Calibration problem 1: the mean relative error

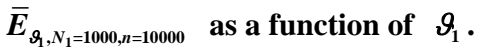

\begin{tabular}{cc}
\hline$\vartheta_{1}$ & $\bar{E}_{\vartheta_{1}, N_{1}=100, n=10000}$ \\
\hline 0.01 & 0.104 \\
0.05 & 0.141 \\
0.1 & 0.170 \\
\hline
\end{tabular}


and a time increment $\delta t>0$, let $t_{i}=i \delta t$, $i_{\hat{n}}=0,1, \cdots, M$, be a discrete set of observation times. Let $\hat{\xi}_{i}$ be the approximation of a realization of $\xi_{t_{i}}, i=1,2, \cdots, M$, obtained integrating with the explicit Euler method one trajectory of the lognormal SABR model (11)-(14). Let us choose $M=100, \quad \delta t=1$, $\varepsilon=0.1, \quad \rho=-0.2, \quad \xi_{0}=\tilde{\xi}_{0}=1 \quad$ and $\quad v_{0}=\tilde{v}_{0}=0.5$. That is, we want to reconstruct the unknown parameters (76) by solving Calibration problem 2 using as data sample the set of the logarithms of the forward prices/ rates observed at time $t_{i}, i=1,2, \cdots, M=100$, along one trajectory of the lognormal SABR model. The set

$$
\hat{\mathcal{D}}_{2}=\left\{\hat{\hat{\xi}}_{i}, i=1,2, \cdots, 100\right\},
$$

is the sample of synthetic data used to solve Calibration problem 2. The data set $\hat{\mathcal{D}}_{2}$ used in the numerical experiment is available at [15].

Proceeding as discussed in Section 3 using the data set $\hat{\mathcal{D}}_{2}$, the least-squares fit of the first-order Taylor's approximation of $\mathcal{L}_{1}^{*}$ with base point $t=0$ evaluated at $t_{i}, \quad i=1,2, \cdots, 10$, gives $\tilde{v}_{0}^{\text {in }}=0.533$ as initial guess of $\tilde{v}_{0}$. Using the data set $\hat{\mathcal{D}}_{2}$, the least-squares fit of the second-order Taylor's approximation of $\mathcal{L}_{1}^{*}$ with base point $t=0$ evaluated at $t_{i}, i=91,92, \cdots, 100$, gives $\varepsilon^{\text {in }}=0.114$ as initial guess of $\varepsilon$. To obtain an initial guess of the parameter $\rho$ we take advantage of the "a priori" information that in finance the correlation between forward prices/rates and stochastic volatility is usually negative. In fact, as it is easy to understand in the financial markets when the prices go down the volatility goes up and viceversa. Exploiting this fact and the fact that $-1<\rho<1$ the initial guess $\rho^{\text {in }}$ of $\rho$ that we choose is $\rho^{\text {in }}=-0.5$.

Starting from the initial guess $\left(\varepsilon^{\text {in }}, \rho^{\text {in }}, \tilde{v}_{0}^{\text {in }}\right)=(0.114,-0.5,0.533)$ the solution of Calibration problem 2 that uses as data the observations $\hat{\xi}_{i}, \quad i=20,30, \cdots, 80$, of $\hat{\mathcal{D}}_{2}$ is obtained solving the nonlinear least-squares problem (75), (73) using the FMINCON routine of Matlab. Note that in (75) we prefer to use only a subset of the observations of the data sample $\hat{\mathcal{D}}_{2}$ (i.e. $i=20,30, \cdots, 80$, a subset of data corresponding to the central part of the trajectory) to avoid the presence of too many addenda in the objective function (75). In the numerical computation the weights $\omega_{i, j}, \quad i=20,30, \cdots, 80, \quad j=1,2,3,4$, are chosen such that the addenda of (75) are of the same order of magnitude. Using $\hat{\mathcal{D}}_{2}$ as data and $\left(\varepsilon^{\text {in }}, \rho^{\text {in }}, \tilde{v}_{0}^{\text {in }}\right)=(0.114,-0.5,0.533)$ as initial guess the solution of problem (75), (73) found is:

$$
\left(\varepsilon, \rho, \tilde{v}_{0}\right)=\left(\varepsilon^{*}, \rho^{*}, \tilde{v}_{0}^{*}\right)=(0.019,-0.168,0.472) .
$$

The relative $L^{2}$-error of the initial guess $\left(\varepsilon^{i n}, \rho^{\text {in }}, \tilde{v}_{0}^{\text {in }}\right)=(0.114,-0.5,0.533)$ with respect to the "true" solution (76) is 0.551 . The relative $L^{2}$-error of the solution (81) of the least-squares problem with respect to the "true" solution (76) is 0.167 .

Let us point out that the data of Calibration problem 2 are supposed to be prices/rates observed in the financial markets, that is, these data are not affected by noise as the data observed in physics. However, let us study the sensitivity of the solution of Calibration problem 2 found with the numerical procedure proposed with respect to the presence of noise in the data. We add noise to the synthetic data contained in $\hat{\mathcal{D}}_{2}$ and we study the quality of the solution of Calibration problems 2 found as a function of the noise properties. In particular given $\vartheta_{2}>0$ let us consider the following "noisy" data sample:

$$
\hat{\mathcal{E}}_{2}=\left\{\hat{\hat{\xi}}_{i}\left(1+\vartheta_{2}(1-2 \text { rand })\right), i=1,2, \cdots, 100\right\},
$$

where rand is a random entry taken from a uniform distribution on the interval $(0,1)$.

Given $\vartheta_{2}>0$ we compute the relative $L^{2}$-error between the "true" solution (76) and the solution of Calibration problem 2 found with the numerical procedure described above. The entire procedure is repeated $N_{2}$ times and the mean of the $\mathrm{L}^{2}$-relative errors of the $\mathrm{N}_{2}$ solutions found is calculated. Let $\bar{E}_{\vartheta_{2}, N_{2}}$ be this mean relative error. The mean relative errors $\bar{E}_{\vartheta_{2}, N_{2}=1000}$ obtained when $N_{2}=1000$ and $\vartheta_{2}=0.01,0.05,0.1$ are shown in Table 3.

Table 3 shows mean relative errors greater than the corresponding mean relative errors of the first numerical experiment. The calibration problem studied in the second experiment is more difficult than the calibration problem studied in the first one. This is due to the quantity of the data and above all to the quality of the quantities entering in the nonlinear least-squares problem deduced from the data sample $\hat{\mathcal{D}}_{2}$ when compared, for example, to the quantity of data contained in the data sample $\hat{\mathcal{D}}_{T=1, n=1000}$ and $\hat{\mathcal{D}}_{T=100, n=1000}$. That is, there are 100 data in $\hat{\mathcal{D}}_{2}$ and 2000 data in $\hat{\mathcal{D}}_{T=1, n=1000}$, $\hat{\mathcal{D}}_{T=100, n=1000}$ and the moments estimated from the data contained in $\hat{\mathcal{D}}_{T=1, n=1000}, \hat{\mathcal{D}}_{T=100, n=1000}$ using formula (71) are of high quality due to the average over a sample of $n=1000$ observations. There is no a similar effect in

Table 3. Calibration problem 2: the mean relative error $\bar{E}_{\vartheta_{2}, N_{2}=1000}$ as a function of $\vartheta_{2}$.

\begin{tabular}{cc}
\hline$\vartheta_{1}$ & $\bar{E}_{\vartheta_{2}, N_{2}=1000}$ \\
\hline 0.01 & 0.239 \\
0.05 & 0.265 \\
0.1 & 0.369 \\
\hline
\end{tabular}


Calibration problem 2.

In the context of finance the natural way of formulating a new version of Calibration problem 2 (i.e. a "single trajectory" calibration problem) that defines more accurately the model parameters is to acquire at the observation times not only the forward prices/rates data, but also the data relative to the prices of one or of several options having as underlying the forward prices/rates. This last problem is a "single trajectory" calibration problem that exploits more deeply than Calibration problem 2 the information contained in the prices. We do not consider this problem here. For example, when the Heston model is used instead of the lognormal SABR model this problem has been studied in [6].

Note that the FMINCON routine of Matlab used to solve problems (72), (73) and (75), (73) is an elementary local minimization routine. Higher quality results can be obtained solving problems (72), (73) and (75), (73) using global minimization methods. Moreover, the explicit moment formulae that define the objective functions (72), (75) can be used to develop ad hoc minimization algorithms to solve problems (72), (73) and (75), (73). This is beyond our purposes in this paper.

In the third numerical experiment we consider a time series of exchange rates between currencies (euro/U.S. dollar exchange rates) in the period going from September 14th, 2010, to July 20th, 2011. The exchange rates considered are daily exchange rates expressed in U.S. dollars and are the closing value of the day (in New York) of one euro expressed in U.S. dollars. Recall that a year is made of about 252 trading days and that a month is made of about 21 trading days. Figure 1 shows the euro/U.S. dollar currency's exchange rate as a function of time. The data set shown in Figure $\mathbf{1}$ is available at [15].

We use the lognormal SABR model to interpret the data shown in Figure 1. In order to use the model in the form (11)-(14) we take the logarithm of the data shown in Figure 1. That is, we solve the Calibration problem 2 using a window of 20 consecutive observations as data and we study the stability of the solution found with respect to shifts of the data along the time series. The model resulting from the calibration can be used to fore-

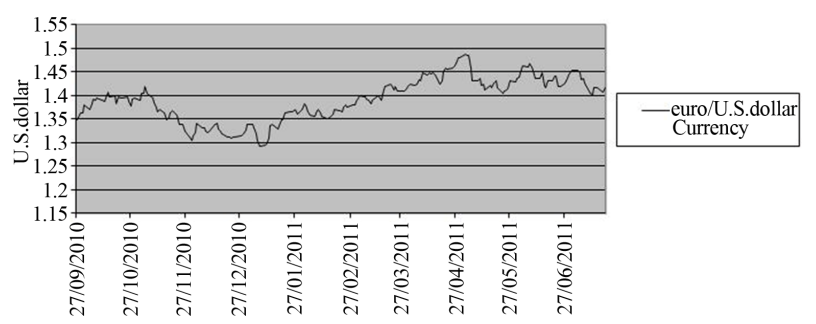

Figure 1. euro/U.S. dollar currency's exchange rate versus time. cast exchange rates and to compute option prices on exchange rates.

We solve Calibration problem 2 using the real data of Figure 1 associated to a time window made of $M+1=20$ consecutive observation times, that is the observations corresponding to 20 consecutive trading days, and we move this window across the data set discarding the datum corresponding to the first observation time of the window and inserting the datum corresponding to the next observation time after the window. The calibration problem (75), (73) is solved for each choice of the data window. We choose $\delta t=1 / 252, \quad \xi_{0}=\tilde{\xi}_{0}$ equal to the first observation (i.e. logarithm of the exchange rate observed) of the window considered, $\omega_{i, j}=1, \quad i=1,2, \cdots, 19, \quad j=1,2,3,4$. The initial guess of the numerical method used to solve the nonlinear least-squares problem (75), (73) has been chosen as follows: $\left(\varepsilon^{i n}, \rho^{i n}, \tilde{v}_{0}^{i n}\right)=(0.05,-0.05,0.05)$. Note that a data window made of twenty data has too few points to implement satisfactorily the asymptotic analysis of the moment formulae discussed in Section 3. Note that to make possible the effective numerical solution of problem (75), (73) the independent variables in (75), (73) have been rescaled.

The reconstructions of the parameters obtained moving the data window along the data set of Figure $\mathbf{1}$ are shown in Figure 2. In Figure 2 the abscissa corresponds to the data window used to reconstruct the model parameters. The data windows are numbered in ascending order beginning with one according to the order in time of the first day of the window considered. In particular, Figure 2 shows that the parameters $\varepsilon, \rho$ reconstructed remain essentially stable when the window is moved along the data time series. Occasionally $\varepsilon$ and $\rho$ have spikes that probably indicate that the numerical procedure used to solve problem (75), (73) has failed.

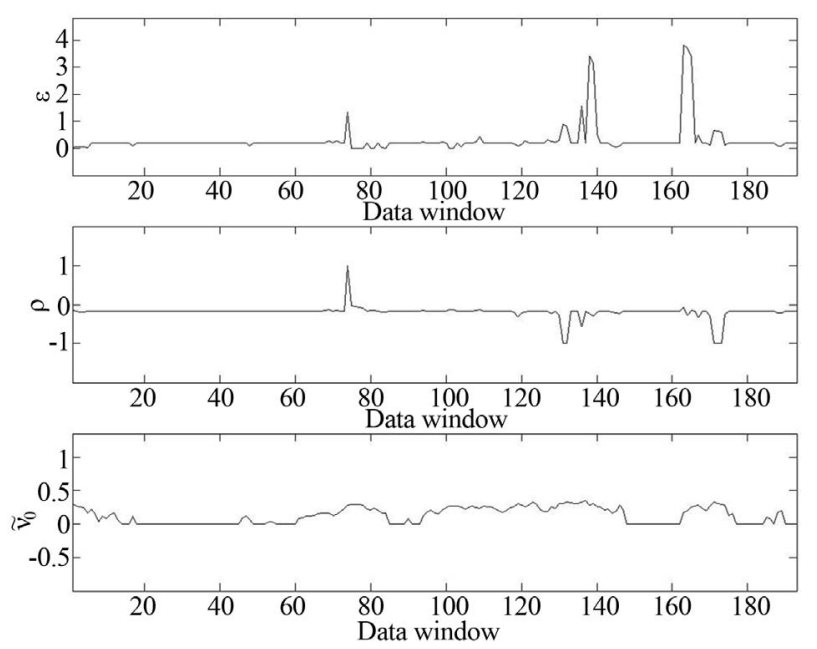

Figure 2. The parameters $\varepsilon, \rho, \tilde{v}_{0}$ reconstructed from the data of Figure 1 versus time. 
The parameter $\tilde{v}_{0}$ reconstructed changes when the window is moved along the data time series. This is correct since $\tilde{v}_{0}$ is the stochastic volatility of the first day of the window and in a stochastic volatility model (such as the lognormal SABR model) there is no reason to expect this value to be constant.

The fact that the values of the parameters $\varepsilon, \rho, \tilde{v}_{0}$, obtained calibrating the lognormal SABR model on the real data shown in Figure 1 through the "moving window" procedure are "stable" (see Figure 2) supports the idea that the lognormal SABR model is able to interpret the data considered. In fact, this stability property suggests that the relation between the data and the parameters of the lognormal SABR model established solving problem (75), (73) is not an artefact of the numerical methods used to solve the nonlinear least-squares problem. In particular, the stability shown guarantees that the lognormal SABR model provides "stable” option prices and hedging strategies.

\section{REFERENCES}

[1] P. S. Hagan, D. Kumar, A. S. Lesniewski and D. E. Woodward, “Managing Smile Risk,” Wilmott Magazine, 2002, pp. 84-108. http://www.wilmott.com/pdfs/021118_smile.pdf

[2] J. C. Cox, J. E. Ingersoll and S. A. Ross, "A Theory of the Term Structure of Interest Rates,” Econometrica, Vol. 53, No. 2, 1985, pp. 385-407.

[3] F. Black and M. Scholes, “c,” Journal of Political Economy, Vol. 81, No. 3, 1973, pp. 637-659.

[4] L. Fatone, F. Mariani, M. C. Recchioni and F. Zirilli, "The Use of Statistical Tests to Calibrate the Normal SABR Model," Journal of Inverse and Ill-Posed Problems, Vol. 21, No. 1, 2013, pp. 59-84. http://www.econ.univpm.it/recchioni/finance/w15

[5] S. Mergner, "Application of State Space Models in Finance,” Universitätsverlag Göttingen, Göttingen, 2004.
[6] F. Mariani, G. Pacelli and F. Zirilli, "Maximum Likelihood Estimation of the Heston Stochastic Volatility Model Using Asset and Option Prices: An Application of Nonlinear Filtering Theory," Optimization Letters, Vol. 2, No. 2, 2008, pp. 177-222.

http://www.econ.univpm.it/pacelli/mariani/finance/w1.

[7] L. Fatone, F. Mariani, M. C. Recchioni and F. Zirilli, "Maximum Likelihood Estimation of the Parameters of a System of Stochastic Differential Equations That Models the Returns of the Index of Some Classes of Hedge Funds," Journal of Inverse and Ill-Posed Problems, Vol. 15, No. 5, 2007, pp. 329-362. http://www.econ.univpm.it/recchioni/finance/w5

[8] L. Fatone, F. Mariani, M. C. Recchioni and F. Zirilli, "Some Explicitly Solvable SABR and Multiscale SABR Models: Option Pricing and Calibration,” Journal of Mathematical Finance, Vol. 3, No. 1, 2013, pp. 10-32. http://dx.doi.org/10.4236/jmf.2013.31002 http://www.econ.univpm.it/recchioni/finance/w14

[9] A. Erdelyi, W. Magnus, F. Oberhettinger and F. G. Tricomi, "Higher Trascendental Functions," McGraw-Hill Book Company, New York, 1953.

[10] J. Hull and A. White, "The Pricing of Options on Assets with Stochastic Volatilities,” The Journal of Finance, Vol. 42, No. 2, 1987, pp. 281-300. http://dx.doi.org/10.1111/j.1540-6261.1987.tb02568.x

[11] B. A. Surya, "Two-Dimensional Hull-White Model for Stochastic Volatility and Its Nonlinear Filtering Estimation,” Procedia Computer Science, Vol. 4, 2011, pp. 1431-1440. http://dx.doi.org/10.1016/j.procs.2011.04.154

[12] A. Erdelyi, W. Magnus, F. Oberhettinger and F. G. Tricomi, “Tables of Integral Transforms,” McGraw-Hill Book Company, New York, 1954.

[13] P. L. Lions and M. Musiela, "Correlations and Bounds for Stochastic Volatility Models,” Annales de l'Institut Henri Poincaré (C) Analyse Non Linèaire, Vol. 24, No. 1, 2007, pp. 1-16.

[14] P. Wilmott, "Paul Wilmott Introduces Quantitative Finance,” Wiley Desktop Editions, Chichester, 2007.

[15] http://www.econ.univpm.it/recchioni/finance/w16 